\title{
The Identification of
}

\section{High Molecular Weight Polynuclear Aromatic Hydrocarbons in a Biologically Active Fraction of Cigarette Smoke Condensate*}

\author{
by M. E. Snook, R. F. Severson, R. F. Arrendale, H. C. Higman, and O. T. Chortyk \\ Tobacco Laboratory, Agricultural Researdh Service, \\ United States Department of Agriculture, Athens, Georgia, U.S.A.
}

\section{INTRODUCTION}

Recent work in this laboratory on the fractionation of cigarette smoke condensate (CSC) for bioassay by mouseback testing has resulted in the isolation of two highly refined polynuclear aromatic hydrocarbon (PAH) subfractions: F-20 and F-55 (Fig. 1) (1-5). Fraction F-20 contained only $0.4 \%$ of the weight of the crude condensate but accounted for virtually all of the tumor-initiating activity of $\operatorname{CSC}(6)$ and "promoted" the development of more tumors than did any previously tested, neutral fraction (7). Using the gel filtration (GF) chromatographic behavior of benzo(a)pyrene on Bio-Beads SX-2, fraction F-20 was further separated

* Presented in part at the 29th Tobacto Chemista' Research Conference, College Park, Md., 1975. into two subfractions F-54 and F-55 (Fig. 2) (5). Fraction F-55 represented only $15 \%$ of F-20 or only $0.05 \%$ of CSC and, more importantly, was almost as tumorigenic as was F-20 (8). In previous work, we isolated the $\mathrm{PAH}$ in $\mathrm{F}-20$ and unambiguously identified the broad spectrum of PAH ranging from indene to indeno(1,2,3-cd)pyrene (9). This paper reports the results of our isolation and identification of the $\mathrm{PAH}$ in the more refined and active F-55 fraction. In effect, GF chromatography on SX-2 resulted in the concentration of the high molecular weight (MW) PAH into a single fraction. We found that F-55 contained only compounds larger than fluoranthene. Thus, the gel filtration step which converted F-20 to F-55 removed all of the low molecular weight PAH and resulted in a concentrated fraction of high molecular weight PAH compounds with considerable biological activity. 
Figure 1. PAH separation scheme for clgarette smoke condensate (CSC).

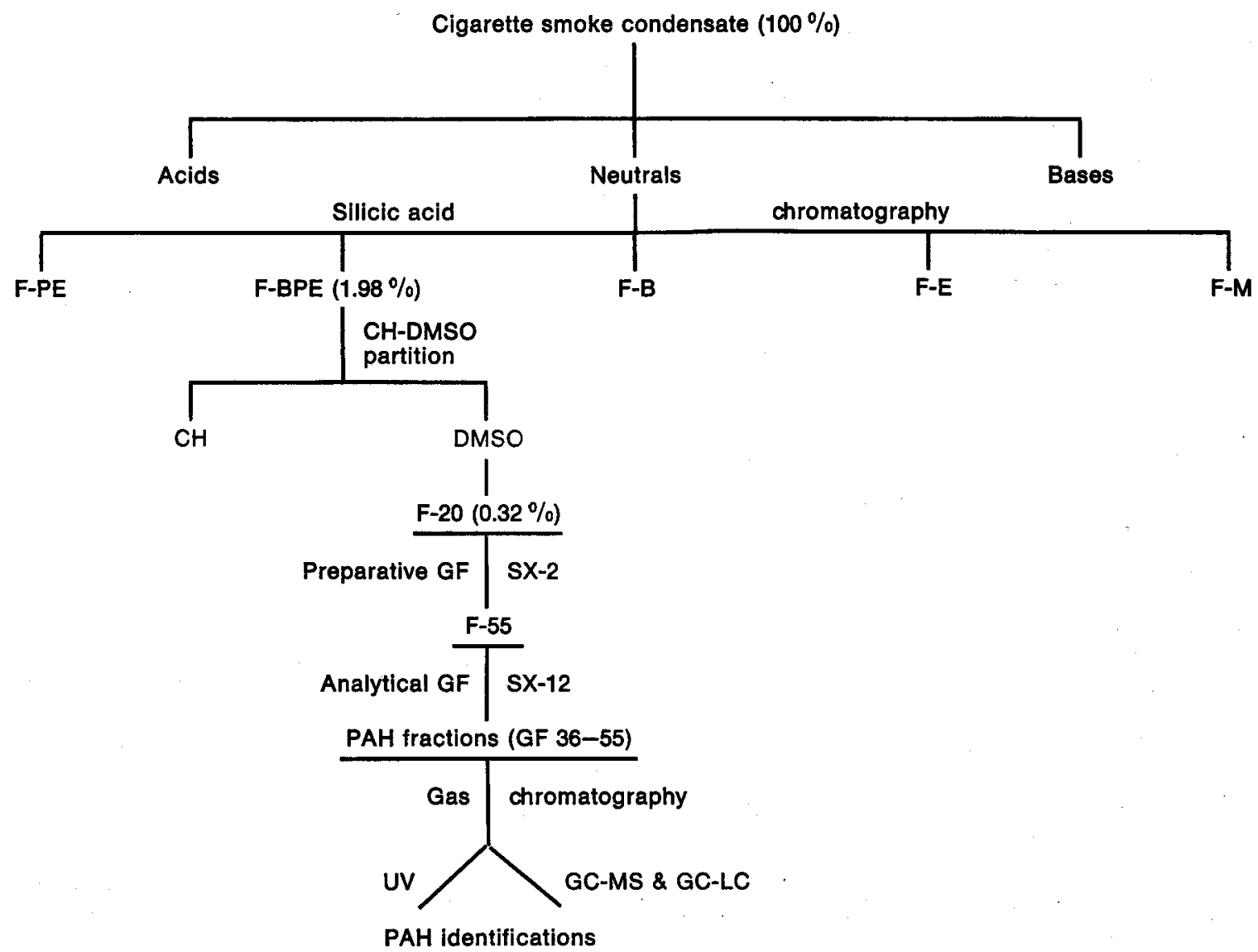

Figure 2. Separation of F-20 on Blo-Beads $\mathbf{S X - 2}$ into F-54 and F-55. Curve A - Elution of benzo(a)pyrene.

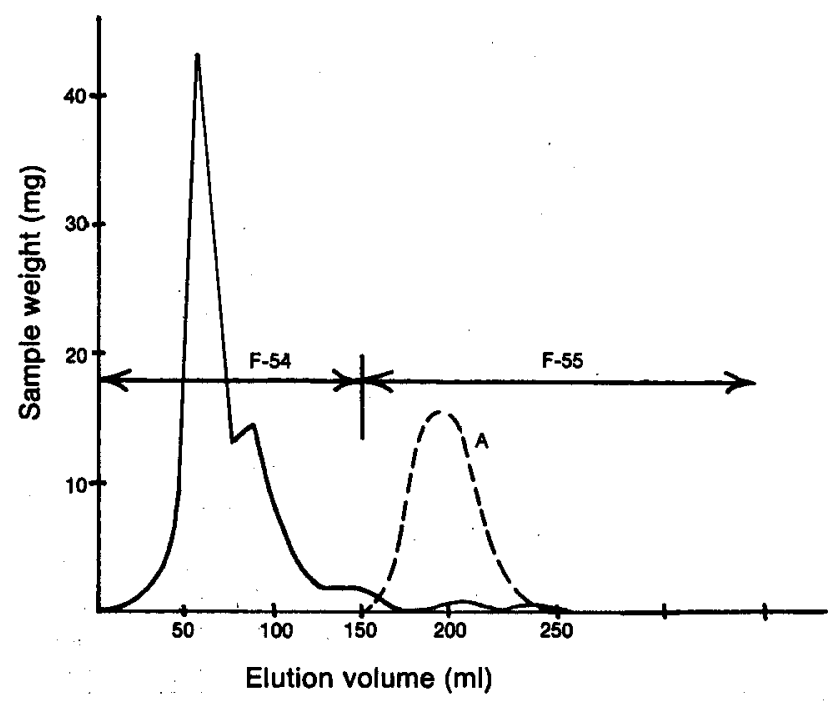




\section{EXPERIMENTAL}

\section{Fractionation of Cigarette Smoke Condensate (CSC)}

All solvents used were Burdick and Jadkson* distilledin-glass grade. The CSC was prepared in $1.0 \mathrm{~kg}$ batches at the Roswell Park Memorial Institute and shipped to us as previously described (10). A total of three $1 \mathrm{~kg}$ batches were fractionated. Although details for the isolation of the PAH concentrate, fraction F-55, have been reported elsewhere (5), the general fractionation scheme is outlined in Figure 1. In brief, CSC neutrals were isolated by consecutive acid and base extractions and then chromatographed on silicic acid. The $25 \%$ benzene : petroleum ether eluate (fraction F$B P E)$ was partitioned between cyclohexane $(\mathrm{CH})$ and dimethylsulfoxide (DMSO) to yield F-20. Fractionation of $3 \mathrm{~kg}$ of CSC yielded $9.6 \mathrm{~g}$ of F-20. Preparative GF of F-20 on Bio-Beads SX-2 in acetone gave $1.0 \mathrm{~g}$ of F-55 (Fig. 2). This highly concentrated PAH fraction was then separated by analytical GF on Bio-Beads SX-12 in benzene on a four-column gel system similar to that described previously (11). The columns $(1.25 \mathrm{~cm}$ $\times 109 \mathrm{~cm}$ ) were connected in series and the slurry was packed in benzene with a total of $200 \mathrm{~g}$ of Bio-Beads SX-12 (dry weight). Samples $(0.25 \mathrm{~g} / \mathrm{ml}$ of F-55 per run) were placed on the columns with a $1 \mathrm{ml}$ injection loop and pumped through the columns at a flow rate of $120 \mathrm{ml} / \mathrm{h}$. Column effluent was monitored at $280 \mathrm{~nm}$ with a Chromatronix Model 230 UV detector, equipped with a flow cell, and $8 \mathrm{ml}$ fractions were collected. Elution of UV-absorbing material began with GF fraction 24 and continued up to GF fraction 55. By the use of standard 2,3,5-trimethylnaphthalene, the beginning of the PAH elution was found to be GF fraction 36. Four separate runs were required to fractionate the entire sample of F-55. Fractions with the same number, from each run, were combined for subsequent GC. The reproducibility of the described gel system allowed such combinations. The amount of material in latter fractions was increased by combining GF fractions 50 and 51 and GF fractions 52 to 55 inclusive.

\section{Gas Cbromatography (GC)}

GF fractions $45,46,47,48,49,50-51$, and 52-55 were subjected to analytical GC analysis on a HewlettPackard Model 5750 gas chromatograph equipped with a $15^{\circ} \times 1 / 8^{\alpha}$ stainless steel column packed with $5 \%$ Dexsil 300 GC on 100/120 mesh Chromosorb W-AW (temperature program: $200-325^{\circ} \mathrm{C}$ at $2 \% \mathrm{~min}$, after an initial hold at $200{ }^{\circ} \mathrm{C}$ for $5 \mathrm{~min} ; 48 \mathrm{ml} / \mathrm{min} \mathrm{He}$; injection temperature, $290^{\circ} \mathrm{C}$; flame detector, $350^{\circ} \mathrm{C}$ ). A Varian Model 485 electronic integrator was used to determine the areas of $\mathrm{GC}$ peaks.

A Hewlett-Padkard Model 5750 gas chromatograph equipped with a thermal conductivity (TC) detector

\footnotetext{
- Referense to a company or product name does nor imply approval or recommendation by the U.S. Department of Agziculture.
}

was used for preparative GC of the above GF fractions. Preparative GC conditions were identical to those for analytical GC. PAH were collected at the exit port of the TC detector in glass capillary tubing (TC oven temperature, $350^{\circ} \mathrm{C}$ ). Whenever possible, samples were collected during the upslope, top, and downslope of GC peaks to give three cuts of a single peak. The GF fraction number and the corresponding number of preparative collection cuts, respectively, were: GF fraction 45, 120; GF fraction 46, 73; GF fraction 47, 91; GF fraction 48, 68; GF fraction 49, 65; GF fraction 50-51, 76; GF fraction 52-55, 36 (total samples, 529).

\section{Ultraviolet Spectral Data of Preparative GC Samples}

The glass capillary tubes containing the PAH from the above preparative GC runs were rinsed into $0.9 \mathrm{ml}$ cuvettes with $95 \%$ ethanol. Ultraviolet (UV) spectra were obtained with a Beckman Acta C III spectrophotometer.

\section{High-Pressure Liquid Chromatography (HPLC)}

A DuPont 830 liquid dromatograph equipped with a $25 \mathrm{~cm} \times 2.4 \mathrm{~mm}$ DuPont Zorbax ODS column was used to separate the individual components of the preparative GC cuts. A linear solvent gradient of $3 \% / \mathrm{min}$, ranging from $65 \% \mathrm{CH}_{3} \mathrm{OH} / \mathrm{H}_{2} \mathrm{O}$ to $85 \% \mathrm{CH}_{3} \mathrm{OH} / \mathrm{H}_{2} \mathrm{O}$, was employed. The initial flow rate was $0.5 \mathrm{ml} / \mathrm{min}$. However, this decreased to about $0.3 \mathrm{ml} / \mathrm{min}$ during the course of this work, possibly due to column compression or blockage. Increased retention times for components did not affect their separation. A total of 156 preparative GC cuts were selected for analysis by HPLC. This quantity represented 51 of the 59 distinct GC peaks in GF fractions 45 to 55 . The preparative GC cuts were concentrated in tapered test tubes to about $5 \mu 1$ (in $\mathrm{EtOH}$ ) and injected into the liquid chromatograph with anthracene as an internal standard. Elution of the samples was monitored at $254 \mathrm{~nm}$, and separated components were collected in $4 \mathrm{ml}$ vials. UV spectra of the separated components were obtained in $\mathrm{CH}_{3} \mathrm{OH}$ (generally $85 \% \mathrm{CH}_{3} \mathrm{OH}$ ). Eluates corresponding to definable peaks or portions of peaks were collected for each run and over 1500 UV spectra were recorded.

\section{GC-Mass Spectral Data}

A Varian Model 1400 GC instrument was used in conjunction with a DuPont $21-492$ mass spectrometer. The gas chromatograph was equipped with a $10^{\circ} \times 1 / 8^{\circ}$ stainless steel column packed with $5 \%$ Dexsil $300 \mathrm{GC}$ on 100/120 Chromosorb W-AW (injection temperature, $290^{\circ} \mathrm{C}$; flame detector, $350^{\circ} \mathrm{C}$; and $20 \mathrm{ml} / \mathrm{min} \mathrm{He}$ ). GF fractions $45,46,47,48,49,50-51$, and 52-55 were dhromatographed by the use of a temperature program of $2^{\circ} / \mathrm{min}$ from 200 to $325^{\circ} \mathrm{C}$. 
Mass spectral (MS) analyses of the GC effluents were performed as follows. The effluent was split with a $1: 1$ splitter, one half going to the flame ionization detector of the gas chromatograph and the other half to the source area of the mass spectrometer. Before MS analysis, a jet separator at $300^{\circ} \mathrm{C}$ was used to strip helium from the GC effluent. Mass spectra of effluent GC peaks were obtained at a scan rate of $10 \mathrm{~s} / \mathrm{mass}$ decade, a minimal resolution of 1000 , and an ionization potential of $70 \mathrm{eV}$. Mass spectra were taken as often as possible during the elution time of a GC peak to determine mass integrity. The spectra were recorded by a high-speed recording oscillograph and/or an AEI DS-30 computerized data system. MS data were analyzed by both manual and computer-aided techniques. HPLCseparated components of doubtful identity were submitted to probe MS analyses after evaporation of the solvent.

\section{Quantitation of}

\section{Selected High Molecular Weight PAH in CSC}

The amounts of several high molecular weight PAH in CSC were quantitated by our recently developed accelerated PAH analysis method $(11,12)$. Three batches of 270 Kentucky 1R1 Reference cigarettes were smoked and the smoke was collected in dry-ice traps. CSC from each batch was treated as follows. The CSC was rinsed into a $1000 \mathrm{ml}$ separatory funnel with benzene, methanol, and ether (100 $\mathrm{ml}$ of each) and washed with
$\mathrm{H}_{2} \mathrm{O}$. The organic solubles were reduced in volume, chromatographed on silicic acid, and eluted first with petroleum ether followed by $25 \%$ benzene:perroleum ether. The second eluate was evaporated and the residue dromatographed on 2 four-column Bio-Beads SX-12 system in benzene. The beginning of the elution of benzo(a)pyrene $(\mathrm{BaP})$ was used to determine the start of the elution of larger PAH. The combined GF fractions from all three GF runs were pooled and analyzed by GC. After application of detector response corrections, the CSC levels of PAH larger than $\mathrm{BaP}$ were determined relative to the known [2.4 $\mu \mathrm{g} / 100$ cigarettes (11)] BeP/BaP levels (Table 3).

\section{RESULTS AND DISCUSSION}

The PAH-containing GF fractions (41-55) were subjected to analytical GC on Dexsil 300 GC. The GC runs showed definite changing profiles (Figs. 3-5). GF fractions 41-44 contained only small amounts of the same PAH occurring predominantly in GF-45. Subsequently, each $G F$ fraction $(45,46,47,48,49,50-51$, and 52-55) was submitted to preparative GC to give cuts representing single peaks or portions of peaks. UV spectra were obtained for each preparative GC cut. Where possible, multiple cuts were taken of peaks for determination of peak integrity. MS data were obtained on each GF fraction by GC-MS techniques. The UV

Figure 3. Gas chromatograms of PAH constituente In GF tractions 45 and 46.

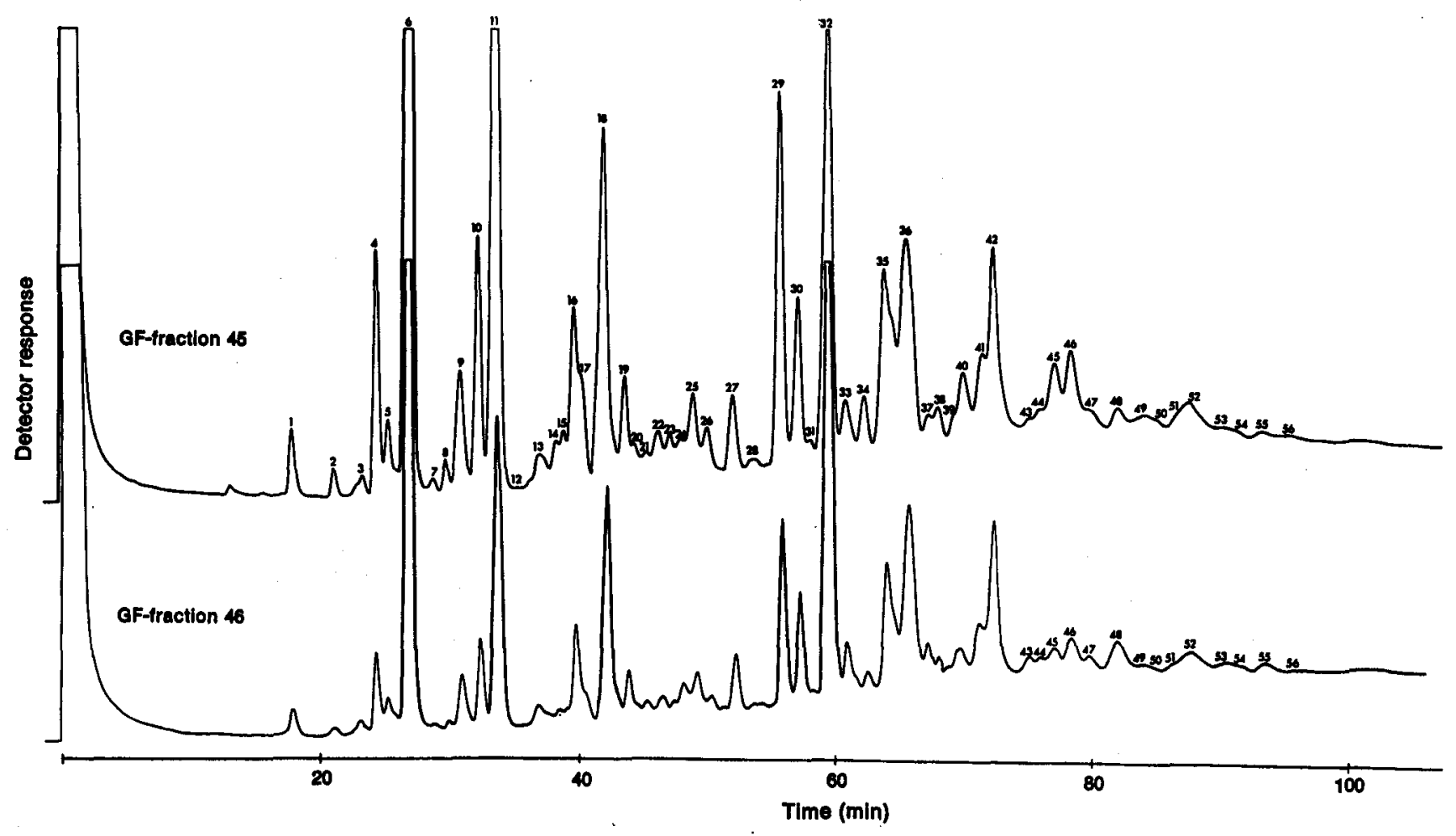


Figure 4. Gas chromatograms of PAH constituents in GF fractions 47 and 48.

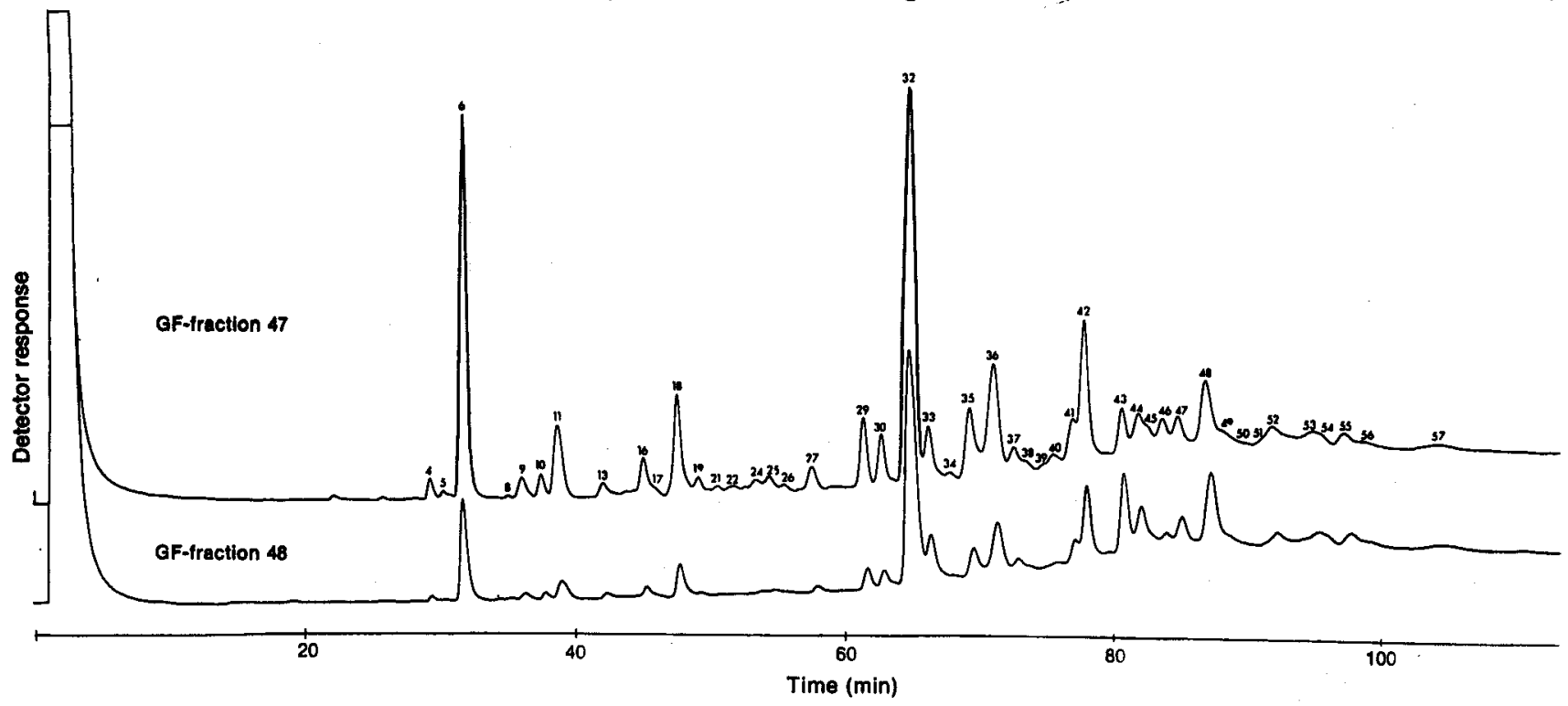

Figure 5. Gas chromatograms of PAH constltuents in GF fractions 49, 50-51 combined, and 52-55 combined.

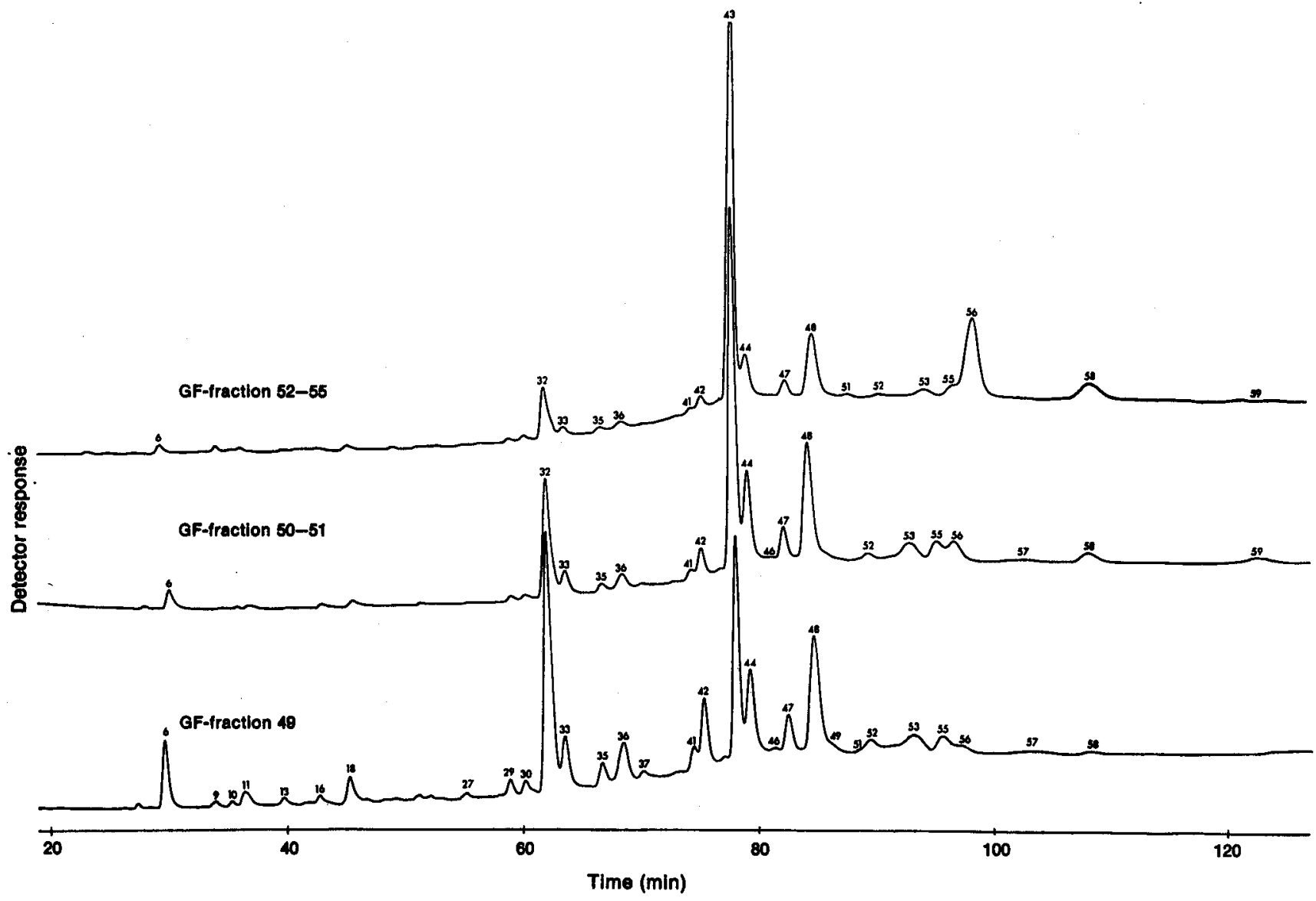

and MS data showed that most GC peaks were complex mixtures. For unambiguous identification, further separation of the components in each peak was necessary. HPLC on Zorbax ODS proved to be an excellent tool in effecting the necessary separations. Individual preparative GC cuts were chromatographed by HPLC, and
UV spectra were obtained on the separated components. In this manner, 51 of the $59 \mathrm{GC}$ peaks in GF fractions $45-55$ were analyzed by HPLC.

The results of the identification and quantitation of the components in GF fractions $45-55$ are given in Table 1. The corresponding gas chromatograms are 
given in Figures 3-5. Peaks are tabulated in order of relative retention time (RRT) with peak number $\$ 32$ $(\mathrm{BaP} / \mathrm{BeP}$ ) being assigned an $\mathrm{RRT}$ value of 1.000 . (Peaks having the same RRT are given the same number in all tables and dromatograms.) Although individual peak percentages will depend on total GC volatiles of a gel fraction, they are strongly indicative of the concentration of components. Where possible, the major component is indicated in multiple component peaks. Applying parent $\mathrm{PAH}$ detector response values, we calculated that at least $80 \%$ of the material in combined GF fractions $45-55$ was GC volatile. The data in Table 1 show that $90-95 \%$ of these GC volatiles were identified. Thus, at least $72 \%$ of the components in the F-55. PAH fraction have now been identified unambiguously.

The basis for identification of most of the compounds in Table 1 is given in Table 2, including the results of the HPLC separations. Usually PAH compounds, whose GC RRT and/or UV spectra are available in the literature, have not been included in Table 2. However, several compounds of this type were included to indicate their HPLC order of elution, given in terms of the RRT. For the most part, the individual RRT can be compared from run to run to indicate order of elution of constituents. However, as work progressed, the RRT of some GC peak components increased, probably due to blockage and the use of the constant pressure pump. Although the separating ability of the HPLC was not affected, a constant flow pump would have produced more consistent RRT. Although analysis time was long by conventional HPLC standards, it proved to be best for preparative separations. Efforts to decrease retention time by increasing the methanol concentration of the mobile phase also decreased resolution. Thus, the use of a $3 \% / \mathrm{min}$ gradient from $85 \%$ to $95 \% \mathrm{CH}_{3} \mathrm{OH} / \mathrm{H}_{2} \mathrm{O}$ halved the RRT of compounds but also compressed the peaks and gave ambiguous UV spectra for collected peaks.

Unambiguous identifications were made by correlation of all the data from the GF, GC, and HPLC separations with the corresponding UV and MS information. The unique properties of the gel columns in retaining and concentrating the high molecular weight $\mathrm{PAH}$ were used to advantage in the final purification step of F-55. The two separation characteristics of the gels have been described (13). Briefly, PAH are separated from interfering material by an absorption-type mechanism and are eluted from the gel columns after the impurities and in order of increasing ring number: Thus, PAH larger than fluoranthene were found in GF fractions 45-55. An additional mechanism of separation also occurs in that methyl PAH eluted from the gels before their parents. An increase of methyl substitution results in earlier elutions relative to parent PAH. These two phenomena are well illustrated by the relative GC peak changes in the gas chromatograms of GF fractions 45-55 (Figs. 3-5) and in the data in Table 1. The dimethyl derivatives of pyrene, chrysene, benzofluoranthene, benzo(a)pyrene, benzo(e)pyrene, indenopyrene, and benzo(ghi)perylene were eluted in earlier gel fractions than their monomethyl analogs, which in turn eluted earlier than the parent compounds. (For brevity, indeno(1,2,3-cd)pyrene will be termed indenopyrene and indeno $\left(1^{\prime}, 2^{\prime}, 3^{\prime}-3,4\right)$ fluoranthene, indenofluoranthene.)

For HPLC on Zorbax ODS, the trend of elution was unsubstituted PAH, followed by monomethyl-, dimethyl-, and trimethyl-substituted PAH. Because of the large number of compounds that co-elute in each gel fraction, it would have been futile to attempt HPLC analysis of the whole gel fraction. Consequently, preparative GC was used to resolve the GF fractions into "peaks" that were collected and then subjected to HPLC. Since PAH differ in their absorption coefficients at $254 \mathrm{~nm}$, the heights of peaks in the HPLC chromatograms, unlike those for GC may not be indicative of the relative concentrations. These separations, in conjunction with the data in Table 2, demonstrated that most of the GC peaks contained multiple components.

Table 1. Composition of gel filtration fractions 45 to 55 .

\begin{tabular}{|c|c|c|c|c|c|c|c|c|c|c|c|c|}
\hline \multirow{3}{*}{$\begin{array}{l}\text { Peak } \\
\text { No. }\end{array}$} & \multirow{3}{*}{ Compounda } & \multirow{3}{*}{ RRTb } & \multicolumn{7}{|c|}{ Gel fraction } & \multirow{2}{*}{\multicolumn{3}{|c|}{$\begin{array}{c}\text { Criteria of } \\
\text { identification }\end{array}$}} \\
\hline & & & 45 & 46 & 47 & 48 & 49 & $50+51$ & $52-55$ & & & \\
\hline & & & \multicolumn{7}{|c|}{ Percent compositionc } & $\begin{array}{l}\text { GC- } \\
\text { RTd }\end{array}$ & UVe & MSt \\
\hline 1 & 4,5-Methylenephenanthrene & 0.362 & 0.82 & 0.26 & 0.21 & 0.07 & - & - & - & + & + & + \\
\hline 2 & Unidentified & 0.415 & 0.36 & 0.15 & 0.12 & 0.05 & - & - & - & & + & + \\
\hline 3 & Dimethylphenanthrene & 0.456 & 0.198 & 0.088 & $<0.058$ & - & - & - & - & & + & + \\
\hline 4 & Fluoranthene & 0.472 & 3.03 & 1.57 & 0.64 & 0.16 & 0.37 & 0.24 & $<0.05$ & + & + & + \\
\hline
\end{tabular}


Table 1 (cont'd.).

\begin{tabular}{|c|c|c|c|c|c|c|c|c|c|c|c|c|}
\hline \multirow{3}{*}{$\begin{array}{l}\text { Peak } \\
\text { No. }\end{array}$} & \multirow{3}{*}{ Compounda } & \multirow{3}{*}{ RRTb } & \multicolumn{7}{|c|}{ Gel fraction } & \multirow{2}{*}{\multicolumn{3}{|c|}{$\begin{array}{c}\text { Criteria of } \\
\text { identification }\end{array}$}} \\
\hline & & & 45 & 46 & 47 & 48 & 49 & $50+51$ & $52-55$ & & & \\
\hline & & & \multicolumn{7}{|c|}{ Percent compositionc } & $\begin{array}{l}\text { GC- } \\
\text { RTd }\end{array}$ & UVe & MSt \\
\hline 5 & Acephenanthrylene & 0.487 & $\begin{array}{c}1.16 \mathrm{~g} \\
\mathrm{M}\end{array}$ & $\begin{array}{c}0.568 \\
M\end{array}$ & $\begin{array}{c}0.088 \\
M\end{array}$ & $\underset{M}{<0.05 g}$ & - & - & - & & + & + \\
\hline 6 & Pyrene & 0.514 & 19.36 & 22.05 & 16.45 & 10.34 & 5.50 & 1.71 & $<0.05$ & + & + & + \\
\hline 7 & Unidentified & 0.540 & 0.12 & $<0.05$ & - & - & - & - & - & & + & + \\
\hline 8 & 8-Methylfluoranthene & 0.558 & 0.36 & 0.07 & 0.16 & $<0.05$ & - & - & - & & +1 & 7) + \\
\hline 9 & $\begin{array}{l}\text { 1-Methylfluoranthene } \\
\text { 2-Methylfluoranthene } \\
\text { 2,3-Benzofluorene } \\
\text { 3,4-Benzofluorene }\end{array}$ & 0.575 & $\begin{array}{r}2.06 \\
M\end{array}$ & $\begin{array}{r}1.20 \\
M\end{array}$ & $\begin{array}{r}0.78 \\
M\end{array}$ & $\begin{array}{r}0.16 \\
M\end{array}$ & $\begin{array}{r}0.42 \\
M\end{array}$ & - & - & + & $\begin{array}{l}+1 \\
+( \\
+ \\
+\end{array}$ & $\begin{array}{l}\text { 7) }+ \\
\text { 7) } \\
+ \\
+ \\
+\end{array}$ \\
\hline 10 & 2-Methylpyrene & 0.597 & 3.42 & 1.72 & 0.80 & 0.26 & 0.39 & 0.14 & $<0.05$ & + & + & + \\
\hline 11 & $\begin{array}{l}\text { 1-Methylpyrene } \\
\text { 4-Methylpyrene }\end{array}$ & 0.618 & $\begin{array}{r}13.20 \\
M \\
M\end{array}$ & $\begin{array}{r}8.00 \\
m \\
M\end{array}$ & $\begin{array}{r}4.08 \\
m \\
M\end{array}$ & $\begin{array}{r}2.31 \\
\mathrm{~m} \\
\mathrm{M}\end{array}$ & $\begin{array}{r}1.05 \\
m \\
M\end{array}$ & $\begin{array}{r}0.47 \\
m \\
M\end{array}$ & $\begin{array}{r}<0.05 \\
\mathrm{~m}\end{array}$ & $\begin{array}{l}+ \\
+\end{array}$ & + & $\begin{array}{l}+ \\
+\end{array}$ \\
\hline 12 & $\begin{array}{l}\text { Methyl-2,3-benzofluorene } \\
\text { Dimethylpyrene } \\
\text { Dimethylfluoranthene }\end{array}$ & 0.639 & $<0.05$ & - & - & - & - & - & - & & $\begin{array}{l}+ \\
+ \\
+\end{array}$ & $\begin{array}{l}+ \\
+ \\
+\end{array}$ \\
\hline 13 & $\begin{array}{l}\text { Methylbenzofluorenes } \\
\text { Dimethylpyrenes } \\
\text { Dimethylfluoranthenes }\end{array}$ & 0.666 & $\begin{array}{c}0.11 \mathrm{~h} \\
\mathrm{M} \\
\mathrm{m} \\
\mathrm{m}\end{array}$ & $\underset{\mathrm{M}}{\mathbf{0 . 3 4 h}}$ & $\begin{array}{c}0.43 \mathrm{~h} \\
\mathrm{M} \\
\mathrm{m} \\
-\end{array}$ & $\begin{array}{c}0.66 \\
\mathrm{M} \\
\mathrm{m} \\
-\end{array}$ & $\begin{array}{r}0.14 \\
M \\
m \\
-\end{array}$ & $\begin{array}{r}<0.05 \\
M \\
- \\
-\end{array}$ & - & & $\begin{array}{l}+ \\
+ \\
+\end{array}$ & $\begin{array}{l}+ \\
\dot{t} \\
+\end{array}$ \\
\hline 14 & $\begin{array}{l}\text { Dimethylpyrene } \\
\text { Benzo(c)phenanthrene }\end{array}$ & 0.689 & $\begin{array}{r}0.32 \\
M \\
t\end{array}$ & $\begin{array}{r}<0.05 \\
M \\
t\end{array}$ & - & - & - & - & - & + & $\begin{array}{l}+ \\
+\end{array}$ & $\begin{array}{l}+ \\
+\end{array}$ \\
\hline 15 & Dimethylpyrene & 0.698 & $\begin{array}{r}0.41 \\
M\end{array}$ & $\begin{array}{r}<0.05 \\
M\end{array}$ & - & - & - & - & - & & + & + \\
\hline 16 & $\begin{array}{l}\text { Benzo(ghi)fluoranthene } \\
\text { Dimethylpyrenes }\end{array}$ & 0.708 & $\begin{array}{r}2.12 \\
M \\
M\end{array}$ & $\begin{array}{r}1.78 \\
M \\
M\end{array}$ & $\begin{array}{r}1.25 \\
M \\
M\end{array}$ & $\begin{array}{r}0.38 \\
M \\
m\end{array}$ & $\begin{array}{r}0.10 \\
M \\
-\end{array}$ & $\begin{array}{r}0.30 \\
M \\
-\end{array}$ & $\begin{array}{r}0.46 \\
M \\
-\end{array}$ & + & + & $\begin{array}{l}+ \\
+\end{array}$ \\
\hline 17 & Dimethylpyrene & 0.719 & 1.11 & 0.46 & 0.15 & $<0.05$ & - & - & - & & + & + \\
\hline
\end{tabular}


Table 1. Composition of gel filtration fractions 45 to 55 (cont'd.).

\begin{tabular}{c|c}
\hline & \\
\hline $\begin{array}{c}\text { Peak } \\
\text { No. }\end{array}$ & Compounda \\
\hline
\end{tabular}

18

1,2-Benzanthracene
Chrysene
Triphenylene
Cyclopenta(cd)pyrene

19

Methyl-2,3-benzofluorene

3,4-Dimethylenepyrene

Dimethylpyrenes

20

Trimethylpyrenes

21

Dimethylbenzofluorene

Methyl-3,4-dimethylenepyrene Trimethylpyrene

22

Methyl-1,2-benzanthracene

Dimethylbenzofluorene

Methylbenzo(ghi)fluoranthene

Trimethylpyrenes

23

3-Methylchrysene

Methyltriphenylene

2-Methylchrysene

Trimethylpyrenes

24

4-Methylchrysene

Methyl-1,2-benzanthracenes

Trimethylpyrene

4,5-Methylenetriphenylene

1-Methylchrysene

6-Methylchrysene

Methyl-1,2-benzanthracene

Trimethylpyrene

26

Dimethylchrysene

Dimethyl-1,2-benzanthracene

3,4-Trimethylenepyrene

27

4,5-Methylenechrysene

Dimethyltriphenylene

Dimethylbenzo(ghi)fluoranthene

Dimethyl-1,2-benzanthracene

28

Dimethyltriphenylene

Dimethylchrysene

$7.51 \quad 6.5$

$6.54 \quad 5.33$

Gel fraction

RRTb

\begin{tabular}{|c|c|c|c|c|c|c|}
\hline \multicolumn{7}{|c|}{ Gel fraction } \\
\hline 45 & 46 & 47 & 48 & 49 & $50+51$ & 52-55 \\
\hline
\end{tabular}

Criteria of

identification

\begin{tabular}{l|l|l}
\hline GC- & UVe & MSf \\
\hline
\end{tabular}

$0.768 \quad 1.30 \mathrm{~h} \quad 0.69 \mathrm{~h} \quad 0.29 \mathrm{~h} \quad 0.31 \mathrm{~h} \quad<0.05 \mathrm{~h} \quad-$

$\begin{array}{lllll}M & M & M & M\end{array}$

$0.777 \quad 0.32 \mathrm{~g} \quad 0.13 \mathrm{~g}<0.05 \mathrm{~g}$

$0.790 \quad 0.38 \mathrm{~h} \quad 0.11 \mathrm{~h} \quad 0.08 \mathrm{~h}<0.05 \mathrm{~h}$

$M \quad M \quad M \quad M$

0.807

$0.71 \mathrm{~h}$

$0.14^{\mathrm{h}}<0.05^{\mathrm{h}}$

$\begin{array}{ccc}M & m & - \\ M & M & M\end{array}$

0.820

$0.48<0.05$

0.829

$0.52 \mathrm{~g}$

$0.32 \mathrm{~g}$

0.326

0.288

$\begin{array}{lll}+ & + & + \\ & + & + \\ + & + & + \\ & + & +\end{array}$

0.84

$1.54 \mathrm{~g}$

$\begin{array}{lll}0.67 \mathrm{~g} & 0.32 \mathrm{~g} & 0.30 \mathrm{~g}\end{array}$

0.238

0.862<smiles>[SeH]</smiles>

$\begin{array}{ccc}0.388 & 0.268 & <0.05 \\ t & t & - \\ t & t & - \\ M & M & M\end{array}$

$\begin{array}{ll}+ & + \\ + & +\end{array}$

$\begin{array}{ll}+ & + \\ + & + \\ + & +\end{array}$

+
$+(18)+$
+

$+\quad+$

+
+
+

$\begin{array}{ll}+ & + \\ + & + \\ + & + \\ + & +\end{array}$

$1.35 \mathrm{~g}$

1.38

1.01

0.278 $\begin{array}{ll}+ & + \\ + & + \\ + & +\end{array}$

+
+
$+(19)+$

+
+ 
Table 1 (cont'd.).

\begin{tabular}{|c|c|c|c|c|c|c|c|c|c|c|c|c|}
\hline \multirow{3}{*}{$\begin{array}{l}\text { Peak } \\
\text { No. }\end{array}$} & \multirow{3}{*}{ Compounda } & \multirow{3}{*}{ RRTb } & \multicolumn{7}{|c|}{ Gel fraction } & \multirow{2}{*}{\multicolumn{3}{|c|}{$\begin{array}{l}\text { Criteria of } \\
\text { identification }\end{array}$}} \\
\hline & & & 45 & 46 & 47 & 48 & 49 & $50+51$ & $52-55$ & & & \\
\hline & & & \multicolumn{7}{|c|}{ Percent compositione } & $\begin{array}{l}\text { GC- } \\
\text { RTd }\end{array}$ & UVe & MSt \\
\hline 29 & $\begin{array}{l}\text { Benzo(b)fluoranthene } \\
\text { Benzo(j)fluoranthene } \\
\text { Benzo(k)fluoranthene }\end{array}$ & 0.946 & $\begin{array}{c}5.27 \mathrm{~h} \\
\mathrm{M} \\
\mathrm{t}\end{array}$ & $\underset{\mathrm{m}}{\mathrm{M}}$ & $\begin{array}{c}2.83^{h} \\
M \\
t\end{array}$ & $\begin{array}{r}1.70 \\
M \\
\mathrm{~m}\end{array}$ & $\begin{array}{r}0.63 \\
M \\
\mathrm{~m}\end{array}$ & $\underset{\mathrm{m}}{0.53}$ & $\begin{array}{r}0.32 \\
\mathbf{m} \\
\mathrm{m}\end{array}$ & + & $\begin{array}{l}+ \\
+(19) \\
+(19)\end{array}$ & $\stackrel{+}{+}+$ \\
\hline 30 & $\begin{array}{l}\text { Benzo(a)fluoranthene } \\
\text { Dimethyl-1,2-benzanthracene }\end{array}$ & 0.966 & $\begin{array}{r}2.63 \\
M \\
t\end{array}$ & $\begin{array}{r}2.86 \\
M\end{array}$ & $\begin{array}{r}1.87 \\
M\end{array}$ & $\begin{array}{r}1.19 \\
M \\
t\end{array}$ & $\begin{array}{c}0.58 \\
M \\
-\end{array}$ & $\begin{array}{c}0.51 \\
M \\
-\end{array}$ & $\begin{array}{r}0.32 \\
M \\
-\end{array}$ & & $\begin{array}{l}+(19) \\
+\end{array}$ & + \\
\hline 31 & Tetramethylpyrene & 0.978 & 0.10 & $<0.05$ & - & - & - & - & - & & & + \\
\hline 32 & $\begin{array}{l}\text { Benzo(e)pyrene } \\
\text { Benzo(a)pyrene }\end{array}$ & 1.000 & 8.25 & 17.35 & 25.98 & 30.93 & 26.16 & 11.79 & 6.02 & + & + & $\stackrel{+}{+}$ \\
\hline 33 & $\begin{array}{l}\text { Perylene } \\
\text { Methylbenzo(j)fluoranthene } \\
\text { Methylbenzo(b)fluoranthene }\end{array}$ & 1.018 & $\begin{array}{r}1.06 \\
M\end{array}$ & $\begin{array}{r}1.45 \\
M\end{array}$ & $\begin{array}{r}3.07 \\
M\end{array}$ & $\begin{array}{r}4.32 \\
M\end{array}$ & $\begin{array}{r}4.52 \\
M\end{array}$ & $\begin{array}{r}2.84 \\
M\end{array}$ & $\begin{array}{r}1.11 \\
M\end{array}$ & + & $\begin{array}{l}+ \\
+ \\
+\end{array}$ & $\begin{array}{l}+ \\
+ \\
+\end{array}$ \\
\hline 34 & $\begin{array}{l}\text { Methylbenzo(j)fluoranthene } \\
\text { Methylbenzo(b)fluoranthene } \\
\text { Methylbenzo(a)pyrene }\end{array}$ & 1.039 & 0.96 & 0.33 & 0.24 & 0.08 & 0.09 & - & - & & $\begin{array}{l}+ \\
+ \\
+\end{array}$ & $\begin{array}{l}+ \\
+ \\
+\end{array}$ \\
\hline 35 & $\begin{array}{l}\text { Methylbenzo(e)pyrenes } \\
\text { Methylbenzo(a)pyrene }\end{array}$ & 1.062 & $\begin{array}{r}3.51 \\
M \\
m\end{array}$ & $\begin{array}{r}5.34 \\
\mathrm{M}\end{array}$ & $\begin{array}{r}4.29 \\
\mathrm{~m}\end{array}$ & $\begin{array}{r}3.13 \\
\mathrm{M} \\
\mathrm{m}\end{array}$ & $\begin{array}{r}1.78 \\
\mathrm{M} \\
\mathrm{m}\end{array}$ & $\begin{array}{r}0.71 \\
\mathrm{M}\end{array}$ & $\begin{array}{r}0.60 \\
M \\
m\end{array}$ & & $\stackrel{+}{+}$ & $\begin{array}{l}+ \\
+\end{array}$ \\
\hline 36 & $\begin{array}{l}\text { Methylbenzo(e)pyrenes } \\
\text { Methylbenzo(a)pyrenes } \\
\text { Methylperylenes }\end{array}$ & 1.087 & 4.97 & 7.69 & 7.71 & 5.91 & 4.04 & 1.75 & 1.11 & & $\begin{array}{l}+ \\
+ \\
+\end{array}$ & $\begin{array}{l}+ \\
+ \\
+\end{array}$ \\
\hline 37 & $\begin{array}{l}\text { Methylbenzo(a)pyrene } \\
\text { Methylperylene } \\
\text { Dimethylbenzo(e)pyrene }\end{array}$ & 1.110 & $\begin{array}{r}0.20 \mathrm{~h} \\
\mathrm{M} \\
\mathrm{t}\end{array}$ & $\begin{array}{r}0.97^{\mathrm{h}} \\
\mathrm{M}\end{array}$ & $\begin{array}{c}0.91 \mathrm{~h} \\
\mathrm{t} \\
\mathrm{M} \\
-\end{array}$ & $\begin{array}{c}1.40 \mathrm{~h} \\
\mathrm{t} \\
-\end{array}$ & $\frac{1.08 \mathrm{~h}}{-}$ & $\stackrel{0.28 h}{-}$ & $\underset{-}{<0.05}$ & & $\begin{array}{l}+ \\
+ \\
+\end{array}$ & $\begin{array}{l}+ \\
+ \\
+\end{array}$ \\
\hline 38 & $\begin{array}{l}\text { Methylperylene } \\
\text { Methylbenzo(a)pyrenes } \\
\text { Methylbenzo(e)pyrenes } \\
\text { Dimethylbenzo(e)pyrene }\end{array}$ & 1.121 & $0.16^{\mathrm{h}} \mathrm{h}$ & $0.57^{\mathrm{h}}$ & $0.26 \mathrm{~h}$ & $0.17^{\mathrm{h}}$ & $<0.05$ & - & - & & $\begin{array}{l}+ \\
+ \\
+ \\
+\end{array}$ & $\begin{array}{l}+ \\
+ \\
+ \\
+\end{array}$ \\
\hline 39 & $\begin{array}{l}\text { Indenofluoranthene } \\
\text { Dimethylbenzo(e)pyrenes } \\
\text { Dlmethylperylene }\end{array}$ & 1.143 & $\begin{array}{r}0.13 \\
M \\
m\end{array}$ & $\begin{array}{r}0.18 \\
M \\
\mathrm{~m}\end{array}$ & $\begin{array}{r}0.19 \\
M \\
t\end{array}$ & - & - & - & - & & $\begin{array}{l}+19 \\
+ \\
+\end{array}$ & $\begin{array}{l}+ \\
+ \\
+\end{array}$ \\
\hline
\end{tabular}


Table 1. Composition of gel filtration fractions 45 to 55 (cont'd.).

\begin{tabular}{|c|c|c|c|c|c|c|c|c|c|c|c|c|}
\hline \multirow{3}{*}{$\begin{array}{l}\text { Peak } \\
\text { No. }\end{array}$} & \multirow{3}{*}{ Compounda } & \multirow{3}{*}{ RRTb } & \multicolumn{7}{|c|}{ Gel fraction } & \multirow{2}{*}{\multicolumn{3}{|c|}{$\begin{array}{c}\text { Criteria of } \\
\text { identification }\end{array}$}} \\
\hline & & & 45 & 46 & 47 & 48 & 49 & $50+51$ & $52-55$ & & & \\
\hline & & & \multicolumn{7}{|c|}{ Percent compositione } & $\begin{array}{l}\text { GC- } \\
\text { RTd }\end{array}$ & UVe & MSt \\
\hline
\end{tabular}

40

$\operatorname{Dibenz}(a, j)$ anthracene

Dimethylbenzo(e)pyrenes

Dimethylperylene

Dimethylbenzo(a)pyrenes

41

Dibenz (a,c)anthracene

Dibenz $(a, h)$ anthracene

Dimethylbenzo(e)pyrenes

Dimethylperylene

Dimethylbenzo(a)pyrenes

Picene

Indenopyrene

Dimethylbenzo(e)pyrenes

Dimethylperylene

Dimethylbenzo(a)pyrenes

Trimethylbenzo(e)pyrenes

43

Benzo(ghi)perylene

Methyldibenz(a,c)anthracene

Dimethylbenzo(a)pyrene

Trimethylbenzo(e)pyrene

44

Anthanthrene

Methylindenopyrene

45

Methyldibenz(a,c)anthracene

Methylindenopyrene

Trimethylbenzo(e)pyrene

46

Trimethylbenzo(e)pyrene

Methylindenopyrene

47

Methylindenopyrenes

Methylbenzo(ghi)perylene

48

Dimethylindenopyrene

Methylbenzo(ghi)perylenes

Methylanthanthrenes

49

Methylbenzo(ghi)perylene

Methylanthanthrenes

Dimethylindenopyrene

50

Methylbenzo(ghi)perylene

Methylanthanthrene

Dimethylindenopyrenes
1.158

$\begin{array}{rr}0.98 & 0.53 \\ \mathbf{t} & \mathbf{t} \\ \mathrm{M} & \mathrm{M}\end{array}$

1.183

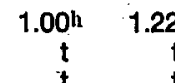

1.201

$\underset{m}{m} \quad 4.53$

6.11

$\begin{array}{rr}6.11 & 5.17 \\ t & t \\ M & M \\ t & - \\ t & - \\ t & -\end{array}$

1.255

$\begin{array}{rr}0.07 \mathrm{~g} & 0.52 \mathrm{~h} \\ \mathrm{M} & \mathrm{M} \\ \mathrm{t} & \mathrm{t} \\ \mathrm{m} & \mathrm{t} \\ \mathrm{t} & \mathrm{t}\end{array}$

$\begin{array}{cc}1.41^{\mathrm{h}} & 6.3 \\ \mathrm{M} & \mathrm{-} \\ - & = \\ - & \end{array}$

6.36
$M$
$=$
$=$

17.05
$M$
-
-

$M$
-
-

43.10

$\begin{array}{rcc}4.27 & 2.44 & 1.16 \\ \mathbf{M} & -\mathrm{M} & -\mathrm{M} \\ \overline{-} & - & - \\ \overline{-} & - & - \\ - & - & -\end{array}$

$\begin{array}{ll}+ & + \\ + & + \\ + & + \\ + & + \\ + & +\end{array}$

1.27<smiles>C1COC1</smiles>

1.52

0.61

2.18

7.35

9.35

6.49

$\begin{array}{llllll}M & M & M & M & M & + \\ t & - & - & - & - & +\end{array}$

1.303

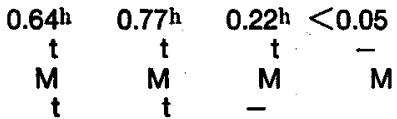

1.332

$0.99 \mathrm{~h}$

$1.11 \mathrm{~h}$

$0.64 h$

0.55

0.27

i

M

M

M

$\bar{M} \quad \bar{M}$

1.360

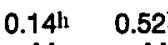

$\begin{array}{cc}0.14 h & 0.5 \\ M & M\end{array}$

$\mathrm{M}^{\mathrm{M}}$

0.76

1.27

1.98

2.03

1.85

1.413

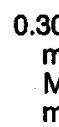

$\begin{array}{ccc}\mathrm{m}_{\mathrm{M}} & 0.98 \mathrm{~h} & 3.22 \\ \mathrm{~m} & \mathrm{M} & \end{array}$

$3.22^{\mathrm{h}}$

$6.50 \mathrm{~h}$

$11.77^{\mathrm{h}}$

$13.41^{\mathrm{h}}$

$9.27 \mathrm{~h}$

1.432

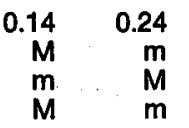

0.36
$\mathrm{~m}$
$\mathrm{M}$
$\mathrm{t}$

0.81
$\mathrm{t}$
$\mathrm{M}$

0.28

$\mathrm{M}$
$\mathrm{M}$

$\mathrm{M}$
$\mathrm{m}$

1.492

$\begin{array}{ccc}0.12 g & 0.12 g & <0.05 \\ M & M & M \\ m & m & M \\ M & M & m\end{array}$

$++$

$\begin{array}{ll}+ & + \\ + & + \\ + & +\end{array}$

+
+
+
+

+
+
+

+
+
+ 
Table 1 (cont'd.).

\begin{tabular}{c|c|c|}
\hline & \\
Peak & & Compounda \\
No. & & \\
\hline
\end{tabular}

51

Dibenzo(b,j)fluoranthene

Methylbenzo(ghi)perylene

Dimethylindenopyrene

Dimethylbenzo(ghi)perylene

52

Dibenzo $(a, e)$ fluoranthene

Dibenzo(a,l)pyrene

Dimethylindenopyrene

Dimethylbenzo(ghi)perylenes

Dimethylanthanthrenes

53

Dibenzofluoranthenes

Dimethylbenzo(ghi)perylene

54

Dibenzofluoranthene

Dimethylbenzo(ghi)perylene

Dimethylanthanthrene

55

Dibenzo(a,e)pyrene

Dimethylbenzo(ghi)perylenes

Trimethylindenopyrene

Trimethylbenzo(ghi)perylene

56

Benzo(b)perylene

Dibenzo(a,i)pyrene

Coronene

Dimethylbenzo(ghi)perylene

Dimethylanthanthrene

Trimethylindenopyrenes

Trimethylbenzo(ghi)perylenes

57

Dibenzo(a,h)pyrene

Dibenzo(e,l)pyrene

Trimethylbenzo(ghi)perylene

58

Methylcoronene

59

Dimethylcoronene

\begin{tabular}{|c|c|c|c|c|c|c|c|c|c|c|}
\hline \multirow{2}{*}{ RRTb } & \multicolumn{7}{|c|}{ Gel fraction } & \multicolumn{3}{|c|}{$\begin{array}{c}\text { Criteria of } \\
\text { identification }\end{array}$} \\
\hline & \multicolumn{7}{|c|}{ Percent compositione } & $\begin{array}{l}\text { GC- } \\
\text { RTd }\end{array}$ & UVe & MSf \\
\hline
\end{tabular}

1.523

$\begin{array}{rr}0.25 & 0.22 \\ m & m \\ m & m \\ M & m\end{array}$

1.545

$\begin{array}{cr}0.53 h & 0.5 \\ \mathbf{M} & \mathbf{m} \\ \mathbf{m} & \mathbf{m} \\ \mathbf{M} & \mathbf{M} \\ \mathbf{m} & \mathbf{M}\end{array}$

1.616

$\begin{array}{rr}0.15 & 0.4 \\ \mathrm{t} & \mathrm{m} \\ \mathrm{M} & \mathrm{M}\end{array}$

1.638

$\begin{array}{rr}0.11 & 0.36 \\ M & M \\ M & M \\ m & m\end{array}$

1.678

$\begin{array}{rr}0.17^{\text {h }} & 0.36 \\ \mathbf{m} & \mathrm{M} \\ \mathbf{t} & \mathrm{m}\end{array}$

1.711

$\begin{array}{rr}- & <0.05 \\ M & m \\ & M \\ m & \text { t } \\ m & m \\ t & t \\ t & t\end{array}$

1.877

$<0.0$
$0.05 h$
$\mathrm{~m}$
$\mathrm{t}$

0.19
$M$
$M$
$t$
$m$
$m$
-

2.49
$M$
$M$
$m$
$m$
$m$
-
-

$0.20^{\mathrm{h}} \quad 0.20 \mathrm{~h}$

$\begin{array}{cc}- & - \\ - & - \\ M & -\end{array}$

$1.11^{\mathrm{h}} \quad 1.22 \mathrm{~h}$

$\mathrm{M}$
$\mathrm{m}$
$\mathrm{m}$

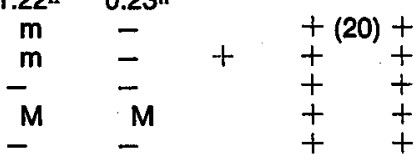

$\begin{array}{rrrrr}79 & 4.31 & 1.71 & & \\ \mathbf{M} & \mathbf{M} & \mathbf{M} & + & + \\ \mathbf{m} & \mathbf{t} & \mathbf{t} & + & +\end{array}$

0.70

$M$
$M$

$0.76 \mathrm{~h}$

$1.41 \mathrm{~h}$

$1.72^{h}$

$\begin{array}{rrrrr}4.06 & 2.36 \\ \mathbf{M} & \mathbf{M} & + & + & + \\ \mathrm{t} & \mathrm{t} & & + & + \\ - & - & & + & + \\ - & - & & + & +\end{array}$

$\begin{array}{rrr}0.98 & 3.62 & 16.68 \\ M & M & m \\ M & M & m \\ M & M & M \\ m & - & - \\ - & - & - \\ - & - & - \\ - & - & -\end{array}$

$\begin{array}{cccc}16.68 & & & \\ m & & +(19) & + \\ m & + & + & + \\ M & + & + & + \\ - & & + & + \\ - & & + & + \\ - & & + & + \\ - & & + & +\end{array}$

0.05 h

$\mathrm{M}$
$\mathbf{m}$

$+\quad+$
$+(19)+$
$+\quad+$

$2.015 \cdots \quad<0.05 \quad 0.24^{h} \quad 0.83^{h} \quad 0.36^{h} \quad 2.19 \mathrm{~h} \quad 5.42^{h}$

$0.50 \mathrm{~h}$

$0.83 h$

a: Whenever possible with multiple component GC peaks, the following designations are used under gel fraction number: M - major component, greater than $30 \%$ of composition; $m$ - minor component, less than $30 \%$ of composition; $t$ trace amount, less than $10 \%$ of composition.

b: Relative to benzo(a)pyrene; a factor of 70.8 converts RRT to minutes from point of injection.

c: Based on total GC volatiles in gel traction assuming unitary detector response.

d: GC retention time identical to standard.

e: UV spectra identical to standard, identical to. literature (reference given), or analogous to parent compound.

f: Molecular ion and fragmentation pattern correlation.

g: Contains other unidentifled material.

h: Major component(s) unidentlfled.

M: Major component. 
Table 2. Identification dataa.

\begin{tabular}{|c|c|c|c|c|}
\hline $\begin{array}{c}\text { GC } \\
\text { peak } \\
\text { No. }\end{array}$ & HPLC RRTb & Compound & $\lambda_{\max }^{\mathrm{c}}$ & Mass $(\mathrm{m} / \mathrm{e})^{\mathrm{d}}$ \\
\hline
\end{tabular}

$2 \quad 1.10 \quad$ Unidentified

3

1.39 Dimethylphenanthrene

Unidentified

(possibly dimethylenephenanthrene)

5

1.15 Acephenanthrylene

1.22 Unidentified

(possibly aceanthrylene)

Unidentified

$7 \quad 1.38 \quad$ Unidentified

$\begin{array}{ll}1.17 & \text { 3,4-Benzofluorene } \\ 1.23 & 2,3-\text { Benzofluorene } \\ 1.39 & \text { 1-Methylfluoranthene } \\ & \text { 2-Methylfluoranthene }\end{array}$

12

Methyl-2,3-benzofluorene

Dimethylpyrene

Dimethylfluoranthene

13

$\begin{array}{ll}1.28 & \begin{array}{l}\text { Methyl-2,3-benzofluorene } \\ \text { (probably the 9-methyl isomer) }\end{array} \\ 1.36 & \text { Methyl-1,2 and/or 2,3-benzofluorene } \\ 1.45 & \text { Unidentified } \\ 1.59 & \text { Methyl-1,2 and/or 2,3-benzofluorene } \\ & \text { Dimethylpyrene } \\ & \text { Dimethylfluoranthene } \\ 1.69 & \text { Dimethylpyrene }\end{array}$

$14 \quad 1.75$ Dimethylpyrene

$15 \quad 1.51 \quad$ Dimethylpyrene

16

$\begin{array}{ll}1.65 & \text { Dimethylpyrene } \\ 1.68 & \text { Dimethylpyrene }\end{array}$

$232,238,278,320,335,355,373$, 400,425

$252,278,287,300$

206, 191

250-4B

204

$232,252,260,286,298,317,328$,

$202 e$

345,363

$228,244,271,323$

202

208

$217,228,240-8 B, 272,278,287$, 350,367

218

216

216

216

216

262

230,215

334

230, 215

$239,288,362$

230,215

$252,265,306-10 B, 328,333,342$

230,215

265

230, 215

$233,240,264,274,335$

$216 e$

254,263

230,215

$240,323,337$

230,215

239, 288

230,215

$235,245,263,276,308,321,337$

230,215

$234,243,265,276,308,321,337$

230,215

$234,244,265,276,308,322,337$

230,215

$242,265,276,321,343$

230,215

$242,265,276.5,322,337$

230,215 
Table 2. (cont'd.).

\begin{tabular}{c|c|c|c|c}
\hline $\begin{array}{c}\text { GC } \\
\text { peak } \\
\text { No. }\end{array}$ & HPLC RRTb & Compound & $\lambda_{\max }$ & Mass (m/e)d \\
\hline
\end{tabular}

$17 \quad 1.42 \quad$ Dimethylpyrene

$242,265,276,328,347$

230,215

19

$\begin{array}{ll}1.43 & \text { Methyl-2,3-benzofluorene } \\ 1.46 & \text { Unidentified } \\ 1.49 & \begin{array}{l}\text { Unidentified } \\ \text { (isomer of HPLC 1.46) }\end{array} \\ 1.55 & \text { 3,4-Dimethylenepyrene } \\ 1.65 & \text { Dimethylpyrene } \\ 1.76 & \text { Dimethylpyrene } \\ 1.86 & \text { Dimethylpyrene }\end{array}$

20

$\begin{array}{ll}1.65 & \text { Unidentified } \\ 1.87 & \text { Trimethylpyrene (2 isomers) } \\ 1.92 & \text { Trimethylpyrene }\end{array}$

$21 \quad 1.47 \quad$ Unidentified (pyrene-type - possibly methyl-3,4-dimethylenepyrene)

1.55 Dimethyl-1,2 and/or 2,3-benzofluorene Methyl-3,4-dimethylenepyrene

1.89 Trimethylpyrene

22

1.58 Methyl-1,2-benzanthracene

Dimethyl-1,2 and/or 2,3-benzofluorene

Unidentified (pyrene-type - possibly methyl-3,4-dimethylenepyrene)

1.63

Unidentified

1.74 Methylbenzo(ghi)fluoranthene

1.95 Trimethylpyrene

2.01 Trimethylpyrene

23

$\begin{array}{ll}1.66 & \text { 3-Methylchrysene } \\ 1.71 & \text { Methyltriphenylene } \\ 1.73 & \text { 2-Methylchrysene } \\ 1.88 & \text { Trimethylpyrene } \\ 1.92 & \text { Trimethylpyrene }\end{array}$

256, 266, 275, 285, 306, 319

$253,267,285,299,309,336,361$

$253,267,286,300,311,336,360$

$233,242,265,276,312,327,343$

$242,254,266,277,327,342$

$247,266,278,323,330$

$226,235,242,253,270,276,281$, $295,310,327,343,357,374,394$

$236,245,269,279,325,340,345$

$236,245,267,278,324,339$

$246,267,275,281,326,340$

255,265

234

$245,266,278,326,339$

275,287

253,262

$275,338,344,365,386$

242

$232,245,279,290,333,348$

240

$234,243,266,278,328,344$

$236,245,267,279$

244,229

244,229

242

248,258

242

242

$235,245,279,327,344$

244

$235,244,268,279$

280B, 288

242

280B, 292 
Table 2. Identification dataa (cont'd.).

\begin{tabular}{c|c|c|c|c}
\hline $\begin{array}{c}\text { GC } \\
\text { peak } \\
\text { No. }\end{array}$ & HPLC RRTb & Compound & $\lambda_{\max }^{c}$ & Mass (m/e)d \\
\hline
\end{tabular}

24 (cont'd.)

Trimethylpyrene

Unidentified

25

$\begin{array}{ll}1.61 & \text { 4,5-Methylenetriphenylene } \\ 1.66 & \text { 1-Methylchrysene } \\ & \text { Unidentified } \\ 1.68 & \text { 6-Methylchrysene } \\ 1.79 & \text { Methyl-1,2-benzanthracene } \\ 1.89 & \text { Trimethylpyrene }\end{array}$

26

\begin{tabular}{|c|c|}
\hline 1.60 & Unidentified \\
\hline 1.64 & Unidentified \\
\hline 1.72 & Dimethylchrysene \\
\hline & $\begin{array}{l}\text { Unidentified } \\
\text { (possibly methylene-1,2-benzanthracene) }\end{array}$ \\
\hline & 3,4-Trimethylenepyrene \\
\hline
\end{tabular}

27

$\begin{array}{ll}1.48 & \text { Unidentified } \\ 1.51 & \text { Unidentified } \\ 1.62 & \text { Unidentified } \\ 1.64 & 4,5-M e t h y l e n e c h r y s e n e \\ 1.75 & \text { Unidentified } \\ & \text { (possibly dimethyldimethylenepyrene) }\end{array}$

1.84 Dimethyltriphenylene

Unidentified (possibly cyclopentenopyrene)

1.94 Dimethylbenzo(ghi)fluoranthene

Dimethylchrysene

1.96 Dimethylchrysene

Dimethyl-1,2-benzanthracene

28

Unidentified (pyrene-type)

Dimethyltriphenylene

Dimethylchrysene
$240,246,280 B, 322,345$

244

240

250,257

$240 e$

242

$223,243,248$

$256,242^{e}$

242

288

$235,245,268,279,330,345$

242

244

247,256

$258,264,267$

268

256,241

286

240

242,240

$221,232,242,260,274,284,293$

$258,282,292,295,367,380,402$, 425

$254,252 e$

$250,257,266,280,315,390,410$

$259,268,311$

$240 \mathrm{e}$

$247,263,280,329,344,378,388$

$256 e$

251,260

256

$236,244,268,278,328,345$

242,240

$233,246,278,290$

$254 e$

260, 270

$256 e$

260,270

256

280,290

256

$245,271,282,349,355,368+252$

250,259

256, 241

259,270

256, 241 
Table 2 (cont'd.).

\begin{tabular}{|c|c|c|c|c|}
\hline $\begin{array}{c}\text { GC } \\
\text { peak } \\
\text { No. }\end{array}$ & HPLC RRTb & Compound & $\lambda_{\max }^{e}$ & $\operatorname{Mass}(\mathrm{m} / \theta)^{\mathrm{d}}$ \\
\hline
\end{tabular}

1.74 Benzo(a)fluoranthene 252

1.90 Dimethyl-1,2-benzanthracene $280,290 \quad 256$

2.00 Unidentified $256,263,300,312,360$

$31 \quad$ Tetramethylpyrene

spectra too weak

1.66 Perylene
1.86 Methylbenzo(j)fluoranthene
1.88 Methylbenzo(b)fluoranthene

34

$\begin{array}{ll}1.78 & \text { Methylbenzo(a)pyrene } \\ 1.85 & \text { Methylbenzo(j)fluoranthene } \\ 1.88 & \text { Methylbenzo(j)fluoranthene }\end{array}$

35

$\begin{array}{ll}1.65 & \text { Methylbenzo(e)pyrene } \\ 1.71 & \text { Methylbenzo(e)pyrene } \\ 1.80 & \text { Methylbenzo(a)pyrene }\end{array}$

36

$\begin{array}{ll}1.73 & \begin{array}{l}\text { Methylbenzo(e)pyrene } \\ \text { Methylperylene }\end{array} \\ 1.77 & \begin{array}{l}\text { Methylbenzo(e)pyrene } \\ 1.82 \quad \begin{array}{l}\text { Methylbenzo(a)pyrene } \\ \text { Methylperylene }\end{array}\end{array} \\ \end{array}$

37

$\begin{array}{ll}1.76 & \text { Unidentified (trace) } \\ 1.83 & \text { Unidentified (trace) } \\ 1.90 & \text { Methylbenzo(a)pyrene } \\ & \text { Methylperylene } \\ 2.11 & \text { Dimethylbenzo(e)pyrene }\end{array}$

$255,266,278,290,307,320,335$

253, 409, 429, 434

221, 236, 265, 278, 288, 307, 319,

332

$360,366,380,386$

$255,265,286,296,350,366,386$

$254,406,436$

$250,300,310,357,386,408$

257, 266, 277, 289

$263,286,297,370,390$

$253,370,390,412,438$

$277,289,319,332$

$275,285,309,408$
266

252

266

266

$266 e$

266

266

266

266

266

266

266

266

266

266

$280,266,264$

266

266

280,265 
Table 2. Identification dataa (cont'd.).

\begin{tabular}{|c|c|c|c|c|}
\hline $\begin{array}{l}\text { GC } \\
\text { peak } \\
\text { No. }\end{array}$ & HPLC RRTb & Compound & $\lambda_{\max }{ }^{c}$ & $\operatorname{Mass}(\mathrm{m} / \mathrm{e})^{d}$ \\
\hline \multirow{6}{*}{$\begin{array}{l}38 \\
\text { (cont }\end{array}$} & 1.86 & Methylperylene (carryover) & $246,252,388-92,410,438$ & 266 \\
\hline & & Methylbenzo(a)pyrene & $296,370,388-91$ & 266 \\
\hline & & Methylbenzo(e)pyrene & $279,287,320,335$ & 266 \\
\hline & 1.91 & Methylbenzo(a)pyrene & $254,265,285,297,368,382,388$ & 266 \\
\hline & & Methylbenzo(e)pyrene & $278,286,317 ; 332$ & 266 \\
\hline & 2.06 & Dimethylbenzo(e)pyrene & $278,290,318,333$ & 280,265 \\
\hline \multirow[t]{5}{*}{39} & 1.90 & Indenofluoranthene & & 276 \\
\hline & 2.09 & Dimethylbenzo(e)pyrene & $268,279,291,319,334$ & 280,265 \\
\hline & & Dimethylperylene & $254,410,436$ & 280,265 \\
\hline & 2.22 & Dimethylbenzo(e)pyrene & $267,286,299,365,383-87$ & 280,265 \\
\hline & 2.26 & Dimethylbenzo(e)pyrene & $268,286,298,365,383-87$ & 280,265 \\
\hline \multirow[t]{7}{*}{40} & 1.91 & Dibenz(a,j)anthracene & & 278 \\
\hline & 2.04 & Dimethylbenzo(e)pyrene & $\begin{array}{l}225,237,269,280,291,322 \\
333-35\end{array}$ & 280,265 \\
\hline & 2.12 & Dimethylbenzo(e)pyrene & $238,258,267,280,291,321,333$ & 280,265 \\
\hline & & Dimethylperylene & $258,423,438$ & 280,265 \\
\hline & & Dimethylbenzo(a)pyrene & $267,368,380,388$ & 280,265 \\
\hline & 2.21 & Dimethylbenzo(a)pyrene & $258,266,286,298,367,387$ & 280,265 \\
\hline & 2.25 & Dimethylbenzo(a)pyrene & $267,286,299,365,388$ & 280,265 \\
\hline \multirow[t]{9}{*}{41} & 1.83 & Dibenz $(a, c)$ anthracene & & 278 \\
\hline & 1.88 & Dibenz(a,h)anthracene & & 278 \\
\hline & 1.92 & Unidentified & $258,284,297,336,386,409$ & $2760^{\circ}$ \\
\hline & 1.95 & Unidentified + trace of indenopyrene & 242,269 & $276 e$ \\
\hline & 2.10 & Dimethylbenzo(e)pyrene & $238,259,268,280,291,322,337$ & 280,265 \\
\hline & & Dimethylbenzo(e)pyrene & $238,254,266,280,292,322,336$ & 280,265 \\
\hline & & Dimethylperylene & $254,414,440$ & 280,265 \\
\hline & 2.16 & Dimethylbenzo(a)pyrene & $255,266,287,298,342,359,379$ & 280,265 \\
\hline & 2.20 & Dimethylbenzo(a)pyrene & $255,266,288,299,352,369,389$ & 280,265 \\
\hline \multirow[t]{6}{*}{42} & 1.90 & Picene & & 278 \\
\hline & 1.94 & Indenopyrene & & 276 \\
\hline & 2.10 & Dimethylbenzo(e)pyrene & $238,281,292,314,322,337$ & 280,265 \\
\hline & 2.14 & Dimethylbenzo(e)pyrene & $222,237,283,294,314,328,342$ & 280,265 \\
\hline & & Dimethylperylene & $250,392,412,436,440$ & 280,265 \\
\hline & 2.19 & Dimethylbenzo(a)pyrene & $256,266,287,297,371,390$ & 280,265 \\
\hline
\end{tabular}


Table 2 (cont'd.).

\begin{tabular}{|c|c|c|c|c|}
\hline $\begin{array}{c}\text { GC } \\
\text { peak } \\
\text { No. }\end{array}$ & HPLC RRTb & Compound & $\lambda_{\max }$ & Mass $(\mathrm{m} / \theta)^{\mathrm{d}}$ \\
\hline
\end{tabular}

\begin{tabular}{rlllr}
\multicolumn{2}{l}{$\begin{array}{l}\text { (cont'd.) } \\
\text { (c.23 }\end{array}$} & Dimethylbenzo(a)pyrene & $256,266,287,299,353,370,389$ & 280,265 \\
& & Dimethylbenzo(a)pyrene & $300,375,393$ & 280,265 \\
2.37 & Trimethylbenzo(e)pyrene & $282,292,334$ & 294,279 \\
2.43 & Trimethylbenzo(e)pyrene & $281,293,321,335$ & 294,279
\end{tabular}

43

$\begin{array}{ll}1.89 & \text { Benzo(ghi)perylene } \\ 1.97 & \text { Methyldibenz(a,c)anthracene } \\ 2.02 & \text { Methyldibenz(a,c)anthracene } \\ 2.13 & \text { Unidentified } \\ 2.17 & \text { Dimethylbenzo(a)pyrene } \\ 2.39 & \text { Trimethylbenzo(e)pyrene }\end{array}$

44

2.00 Anthanthrene

2.16 Methylindenopyrene

276, 286

276

275, 286

292, 277

$260,286,297,357$

292, 277

$291,300,360 B, 388$

292, 290

280,265

$281,292,338$

294,279

$\begin{array}{ll}2.04 & \text { Methyldibenz(a,c)anthracene } \\ 2.18 & \text { Unidentified } \\ 2.26 & \text { Methylindenopyrene } \\ 2.45 & \text { Trimethylbenzo(e)pyrene }\end{array}$

46

$\begin{array}{ll}2.10 & \text { Unidentified } \\ 2.35 & \text { Trimethylbenzo(e)pyrene } \\ 2.40 & \text { Methylindenopyrene }\end{array}$

47

\begin{tabular}{|c|c|}
\hline 2.11 & $\begin{array}{l}\text { Unknown (possibly methyl isomer of } \\
\text { peak 46, HPLC RRT 2.10) }\end{array}$ \\
\hline 2.15 & Methylindenopyrene \\
\hline 2.22 & Methylindenopyrene \\
\hline
\end{tabular}

Methylbenzo(ghi)perylene

482.06 Unidentified (possibly methyl isomer of peak 46, HPLC RRT 2.10)

$\begin{array}{ll}2.19 & \begin{array}{l}\text { Dimethylindenopyrene } \\ \text { Methylbenzo(ghi)perylene }\end{array} \\ 2.21 & \text { Methylbenzo(ghi)perylene } \\ 2.25 & \text { Methylanthanthrene } \\ 2.39 & \text { Methylanthanthrene }\end{array}$

\section{5,286}

285

$250,290,302,315,361,375,385$

$280,292,340$

$242,276,284,326,343,358,362$

$292,328,343$

$249,301,314,344,361,377,385$

$242-250,275,285,301,315,356$, $363,374,388$

$250,300,314,358$

$250,275,290,300,314,344,361$. 380,384

$275,288,299,329,346,362,381$, 384

1

$275,285,355+374,388$

$250,299,313,356$

$277,289,300,320,380-85$

$276,288,300,329,350,364$,

382-85

$290,305,406,420,430$

$260,295,308,405,424,428,456$
290,275

306, 391

304

290, 275

292, 277

292

290, 275

294, 279

292, 290 e

294, 279

290, 275

306

290, 275

290,275

290, 275

290, 275

290 
Table 2. Identification dataa (cont'd.).

\begin{tabular}{|c|c|c|c|c|}
\hline $\begin{array}{c}\text { GC } \\
\text { peak } \\
\text { No. }\end{array}$ & HPLC RRTb & Compound & $\lambda_{\max }$ & Mass (m/e)d \\
\hline \multirow[t]{5}{*}{49} & 2.29 & Methylbenzo(ghi)perylene & $288,300,347,363,380-85 B$ & 290 \\
\hline & & Methylanthanthrene & $292,306.5,404,412,421,429,436$ & 290 \\
\hline & 2.38 & Methylanthanthrene & $\begin{array}{l}294,306.5,399,405,412,420 \\
428,436\end{array}$ & 290 \\
\hline & 2.49 & Methylanthanthrene & $293,304,405,420,428,436$ & 290 \\
\hline & 2.73 & Dimethylindenopyrene & $253,292,303,315,363,380-90 B$ & 304 \\
\hline \multirow[t]{6}{*}{50} & 2.48 & Unidentified & $295,305,323,358$ & $286 e$ \\
\hline & 2.64 & Methylbenzo(ghi)perylene & $289,300.5,363,384$ & $290 e$ \\
\hline & & Methylanthanthrene & $292,305,422,429,434$ & $290 e$ \\
\hline & 2.79 & Dimethylindenopyrene & $253,288,300,315,363$ & 304 \\
\hline & 2.81 & Dimethylindenopyrene & $\begin{array}{l}251.5,291,302,315,363 \\
375-85 B\end{array}$ & 304 \\
\hline & 2.86 & Dimethylindenopyrene & $251,291-92,302,315,363,385$ & 304 \\
\hline \multirow[t]{4}{*}{51} & 2.13 & Dibenzo(b,j)fluoranthene & & 302 \\
\hline & 2.51 & Methylbenzo(ghi)perylene & $288,300,363,380-86 B$ & 290 \\
\hline & 2.65 & Dimethylindenopyrene & $251,303,315,363,387$ & 304 \\
\hline & 2.74 & Dimethylbenzo(ghi)perylene & $291,302,364,382,387$ & 304 \\
\hline \multirow[t]{10}{*}{52} & 2.13 & $\begin{array}{l}\text { Unidentified } \\
\text { (probably dibenzo(a,e)fluoranthene) }\end{array}$ & $\begin{array}{l}240,254,264,285,300,316 \\
332(20)\end{array}$ & 302 \\
\hline & 2.16 & $\begin{array}{l}\text { Unidentified } \\
\text { (possibly dibenzo(j,I)fluoranthene) }\end{array}$ & $248,284,303,319,330,343(21)$ & 302 \\
\hline & & Dibenzo(a,l)pyrene & & 302 \\
\hline & 2.22 & $\begin{array}{l}\text { Unidentified } \\
\text { (probably a dibenzofluoranthene) }\end{array}$ & $249,269,280,305,362,382$ & 302 \\
\hline & 2.47 & Dimethylindenopyrene & $251,316,358$ & 304 \\
\hline & & Dimethylbenzo(ghi)perylene & $278,290,301,360,386$ & 304 \\
\hline & 2.53 & Dimethylbenzo(ghi)perylene & $\begin{array}{l}278,290,302,330,350,365,384 \\
387\end{array}$ & 304 \\
\hline & 2.70 & Dimethylanthanthrene & $233,261,297,309,406,439$ & 304 \\
\hline & 2.75 & Dimethylanthanthrene & $263,297,309,438$ & 304 \\
\hline & 2.84 & Dimethylanthanthrene & $234,262,297,310,439$ & 304 \\
\hline \multirow[t]{3}{*}{53} & 2.29 & $\begin{array}{l}\text { Unidentified } \\
\text { (probably a dibenzofluoranthene) }\end{array}$ & $224,227,248,291,362,377,382$ & $302 e$ \\
\hline & 2.68 & Dimethylbenzo(ghi)perylene & $275 B, 291,302,331,367,390$ & 304 \\
\hline & & & $\cdot$ & \\
\hline 54 & 2.15 & $\begin{array}{l}\text { Unidentified } \\
\text { (probably a dibenzofluoranthene) }\end{array}$ & $243,260,270,303,315,399$ & $302 e$ \\
\hline
\end{tabular}


Table 2 (cont'd.).

\begin{tabular}{|c|c|c|c|c|}
\hline $\begin{array}{c}\text { GC } \\
\text { peak } \\
\text { No. }\end{array}$ & HPLC RRTb & Compound & $\lambda_{\max }$ & Mass $(\mathrm{m} / \mathrm{e})^{\mathrm{d}}$ \\
\hline
\end{tabular}

$54 \quad 2.70$ Dimethylbenzo(ghi)perylene

(cont'd.)

Dimethylanthanthrene

55

2.14 Dibenzo(a,e)pyrene

2.18 Unidentified

(probably a dibenzofluoranthene)

2.55 Dimethylbenzo(ghi)perylene

Dimethylbenzo(ghi)perylene

2.67 Dimethylbenzo(ghi)perylene

3.04 Trimethylindenopyrene

56

2.0

2.35

Benzo(b)perylene

Dibenzo(a,i)pyrene

2.59

Coronene

Dimethylbenzo(ghi)perylene

Dimethylanthanthrene

3.18 Dimethylbenzo(ghi)perylene

Dimethylanthanthrene

3.34 Trimethylindenopyrene

Trimethylbenzo(ghi)perylene

3.50 Trimethylindenopyrene

Trimethylbenzo(ghi)perylene

57

$\begin{array}{ll}2.35 & \text { Dibenzo(a,h)pyrene } \\ 2.45 & \begin{array}{l}\text { Unidentified } \\ \text { (possibly dibenzo(e,l)pyrene) }\end{array} \\ 3.00 & \begin{array}{l}\text { Unidentified } \\ \text { (possibly methyldibenzo(a,e)pyrene) }\end{array}\end{array}$

Unidentifled (probably a methyl isomer of peak 55, HPLC RRT 2.28)

Trimethylbenzo(ghi)perylene

Methylcoronene

Unidentified

(probably a methyldibenzopyrene)
$275 B, 291,302,331,369,390$

304

309,433

304

$302 \mathrm{e}$

$282,294,308,344,361,381$

$302 \mathrm{e}$

291, 302, 367, 389

304

$291,302,366,384,389$

304

$292,302,365 \mathrm{~B}, 389 \mathrm{~B}$,

304

253,317

$318 \mathrm{e}$

302

302

300

$292,302,365,383,387$

304

308,436

304

$291,303,368,385$

304

310,435

304

253

318

293, 303, 366B, 386B

318

253

318

$279,292,304,367 B, 389 B$

318

302

$223,274,286,329(19)$

302

$274,286,302$

$316 e$

$272,284,295,309,363,383$

$316 e$

289,302

318

$291,303,325,335,340$

314

316

$291,303,340$

328

330,326

Unidentified

a: Except to indicate HPLC RRT for selected PAH, this table presents identification data for compounds whose GC retention time and/or literature UV data are lacking.

b: Relative to anthracene; a factor of 48 converts HPLC RRT to time in minutes from point of injection (see text for limitations of HPLC RRT).

c: $85 \% \mathrm{CH} \mathrm{OH}_{3} / \mathrm{H}_{2} \mathrm{O}$; $\mathrm{B}$ - broad.

d: Unless otherwise noted, mass obtained by GC-MS techniques.

e: Mass obtained by submitting trapped HPLC peak to probe MS analysis. 
Figure 6. HPLC chromatogram of GC peak number 9. (Anthracene added as internal standard.)

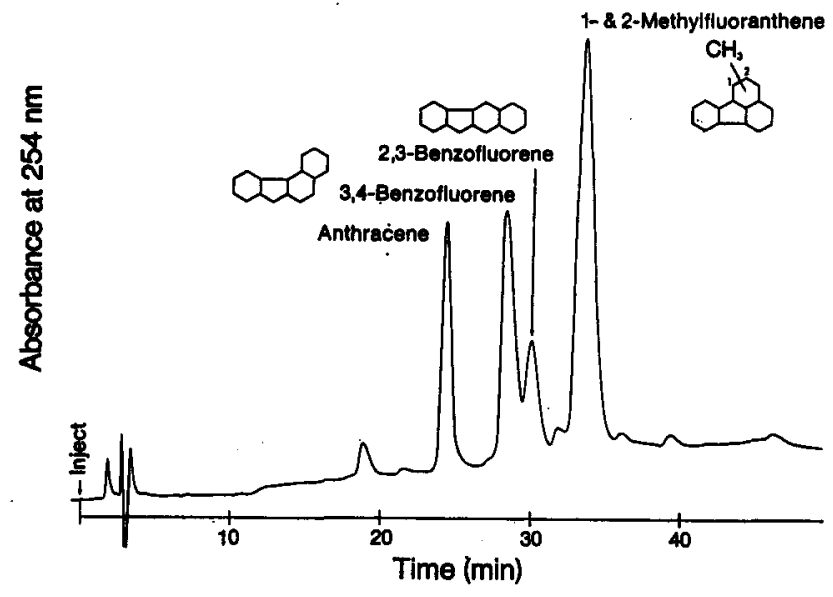

Several examples of the remarkable separations achieved by HPLC are shown in Figures 6-10. The HPLC separations of GC peak number 9 of GF fraction 49 is shown in Figure 6. Resolved compounds were 3,4-benzofluorene, 2,3-benzofluorene, and two methyl fluoranthenes. UV data confirmed that the broadness and asymmetry of the methyl fluoranthene peak was due to the presence of the 1- and 2-methyl isomers. The HPLC separation of GC peak number 33 of GF fraction 45 is given in Figure 7. Perylene, benzo(a)pyrene, and two methylbenzofluoranthenes were separated. The benzo(a)-
Figure 7. HPLC chromatogram of GC peak number 33. (Anthracene added as internal standard.)

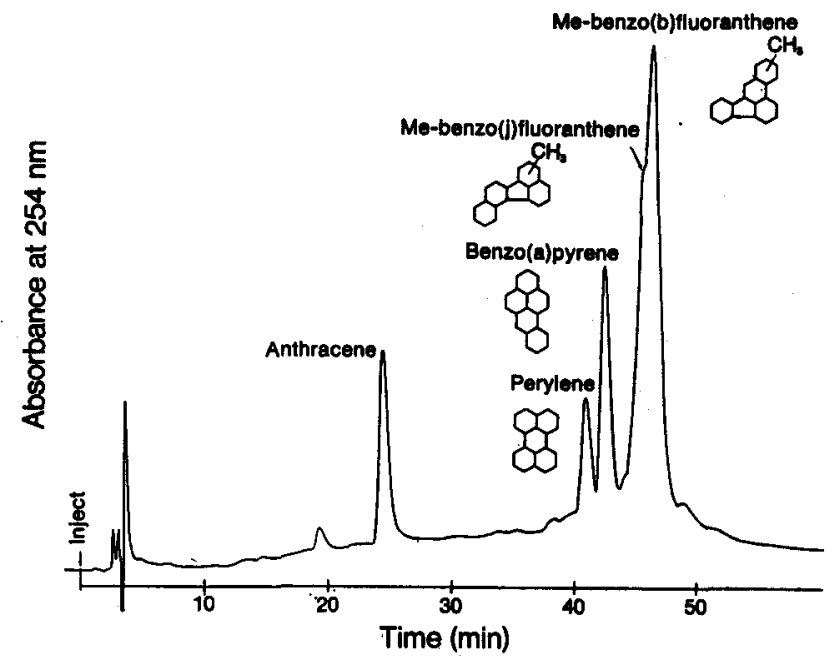

pyrene was a carryover from peak number 32 . Such carryover was found in many cases with the high molecular weight, low volatility PAH. The complexity of some GC peaks is illustrated in Figure 8. HPLC of peak number 41 of GF fraction 45 separated dibenz $(a, c)$ anthracene, dibenz( $\mathrm{a}, \mathrm{h})$ anthracene, a trace of indenopyrene, a dimethylperylene, two dimethylbenzo(e)pyrenes, and two dimethylbenzo(a)pyrenes. HPLC RRT and MS analyses of the two large unknown peaks indicated that they were unsubstituted PAH with m/e of 276 . Lack of literature UV data on several possible candidates

Flgure 8. HPLC chromatogram of GC peak number 41. (Anthracene added as internal stendard.)

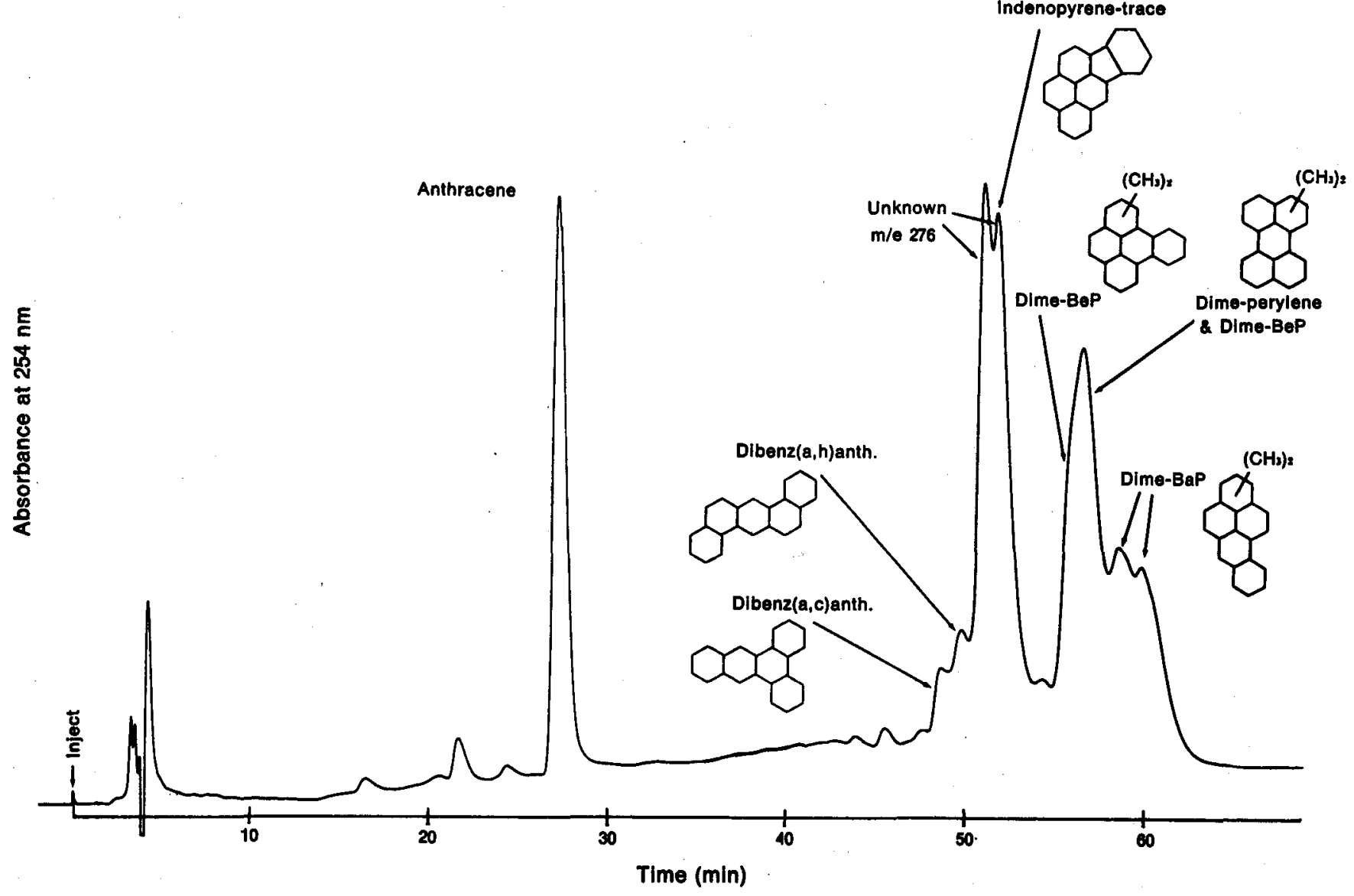


Figure 9. HPLC chromatogram of latter half of GC peak number 42. (Anthracene added as internal standard.)

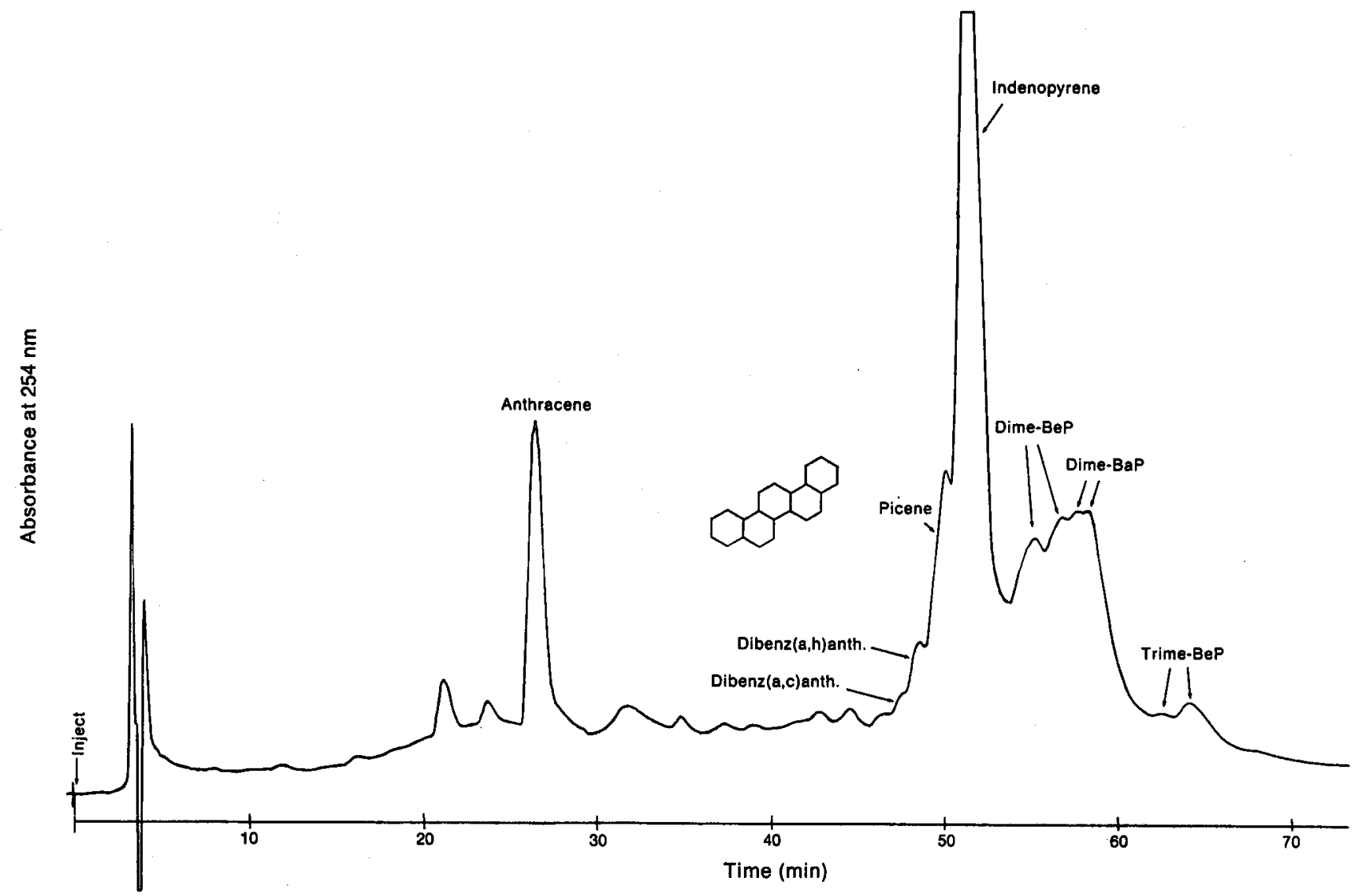

for these peaks prevented conclusive identification. Possible assignments are aceperylene and dibenzo(b,ghi)fluoranthene or dibenzo(b,mno)fluoranthene. The HPLC resolution of the backslope of GC peak number 42 of GF fraction 47 is shown in Figure 9. Identified compounds were: traces of dibenz $(a, c)$ anthracene and dibenz- (a,h)anthracene, picene, indenopyrene, two dimethylbenzo(a)pyrenes, two dimethylbenzo(e)pyrenes, and a trimethylbenzo(e)pyrene. The decreasing quantities of dimethyl and trimethyl derivatives in GF fraction 47 are quite apparent. The final example of the HPLC separations (Figure 10) shows components from GC peak

Figure 10. HPLC chromatogram of GC peak number 44; a - unidentified. (Anthracene added as internal standard.)

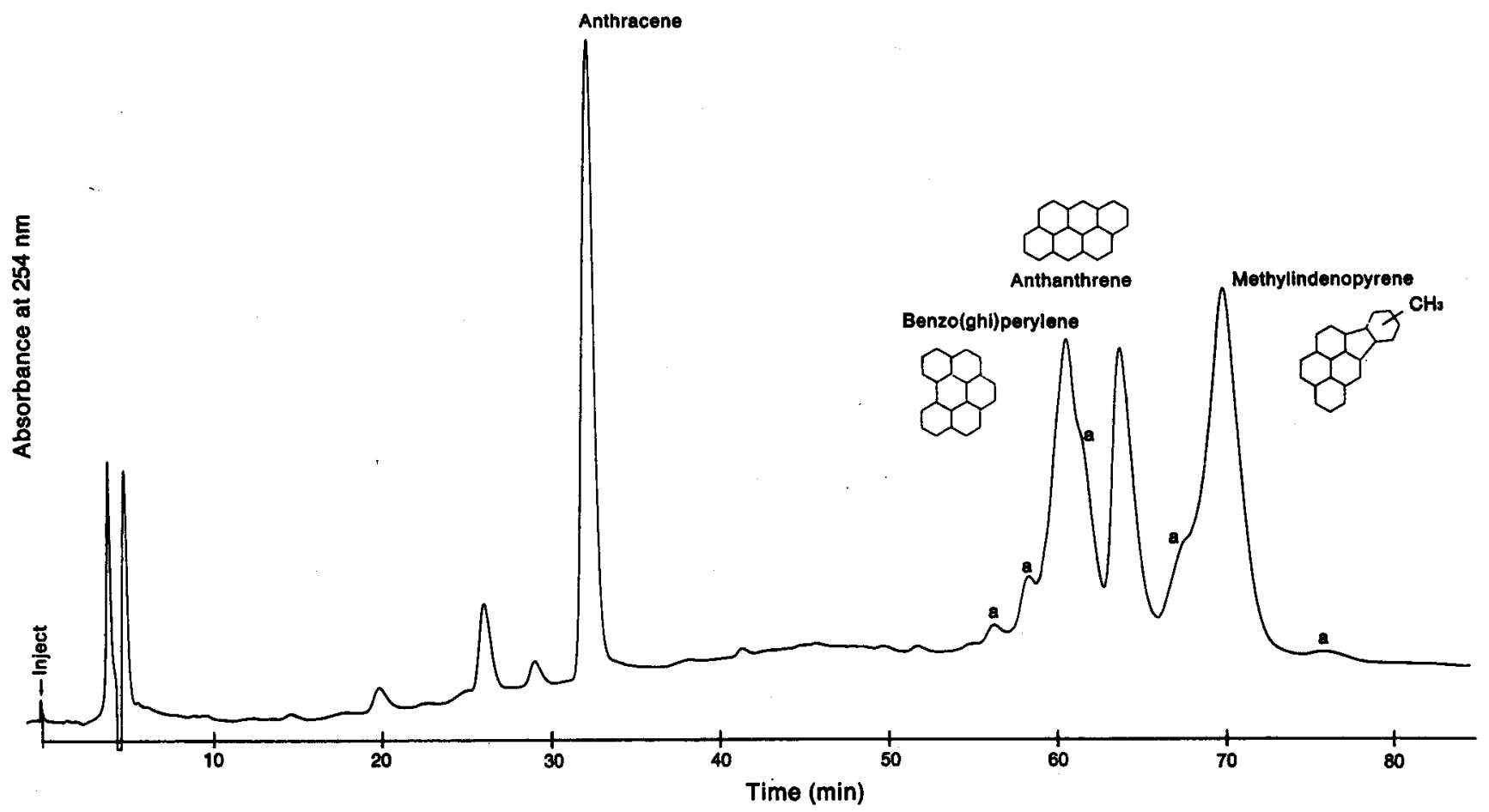


Figure 11. UV spectrum of collected GC peak number 5.

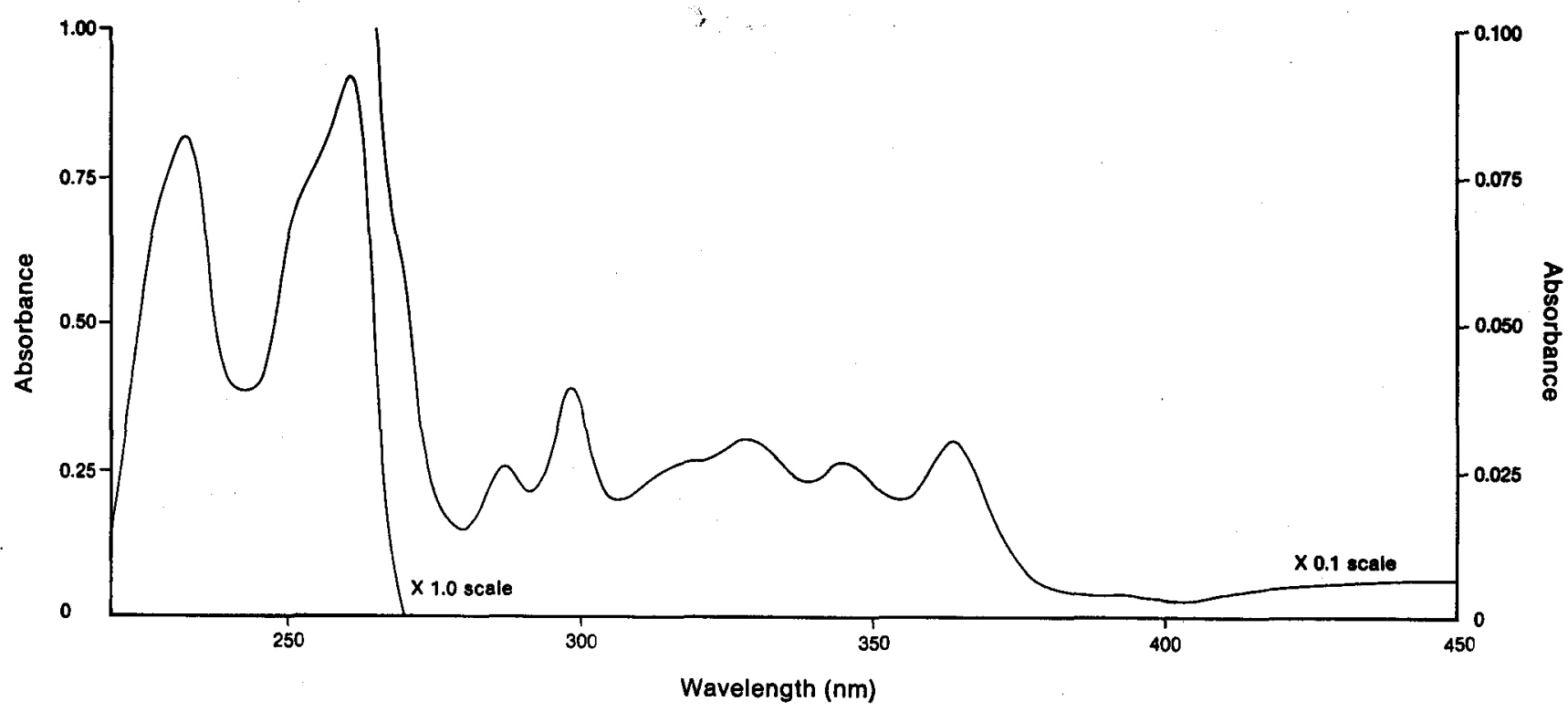

number 44 of GF fraction 47. Benzo(ghi)perylene (carryover from peak number 46), anthanthrene, and methylindenopyrene were identified. Additional data on the above separations can be found in Table 2, under the corresponding GC peak number.

In this study, several new components of CSC were identified. These include 3,4-dimethylenepyrene, 3,4-trimethylenepyrene, cyclopenta(c,d)pyrene, 4,5-methylenetriphenylene, benzo(b)perylene, and several dibenzofluoranthenes. The ladk of available standards and literature UV spectra for many PAH has hindered further identifications. This was particularly true with the dibenzofluoranthene series. For the ten possible dibenzofluoranthenes, only four literature UV spectra could be found.

Some of the difficulties in assigning conclusive identificacations are illustrated by the following case. The UV spectrum of the major component in GC peak number 5 is shown in Figure 11. This compound had a molecular weight of 202 and it was tentatively identified as acephenanthrylene. To our knowledge, the UV spectrum of acephenanthrylene has not been reported; however, acephenanthrylene has been reported to elute from a

Table 3. Levels of selected large ring PAH in clgarette smoke condensate from 1R1 clgarettes.

\begin{tabular}{l|c}
\hline \multicolumn{1}{c|}{ PAH } & $\begin{array}{c}\text { Amounta } \\
(\mu \mathrm{g} / 100 \text { cigarettes })\end{array}$ \\
\hline Benzo(a)pyrene/benzo(e)pyrene & 2.4 \\
Perylene & 0.3 \\
Indenopyrene & 0.6 \\
Benzo(ghi)perylene & 0.5 \\
Anthanthrene & 0.3 \\
Methylbenzo(ghi)perylenes & 0.5 \\
Coronene & 0.1 \\
\hline
\end{tabular}

a: Values obtained using flame lonization detector and employing Internal standard methods. Methyl derivatlves were assumed to yield detector response identical to parent compounds.
SE-30 GC column between fluoranthene and pyrene (14). This seems reasonable as acephenanthrylene is an isomer of fluoranthene.

The levels of several high molecular weight PAH in CSC were determined. For this purpose, 810 research cigarettes were smoked, and the PAH were isolated and quantitated by our accelerated technique $(11,12)$. The results (Table 3 ) show that the level of each of the larger PAH was smaller than that of the benzopyrenes. The data in Table 3 compare favorably with previous results (15).

On the basis of the data in Table 1, we evaluated the relative concentrations of the large $\mathrm{PAH}$ in cigarette smoke. Compounds that occurred in major amounts are: benzofluoranthenes, benzo(a)pyrene, benzo(e)pyrene, perylene, indenopyrene, benzo(ghi)perylene, anthanthrene, coronene, and their methyl and dimethyl derivatives. PAH that occur in minor to trace quantities are dibenzanthracenes, dibenzophenanthrenes, dibenzofluoranthenes, and the dibenzopyrenes. Several dibenzanthracenes, dibenzophenanthrenes, and dibenzopyrenes have been identified in $\operatorname{CSC}(15,16)$. Surprisingly, however, the more abundant methyl and dimethyl derivatives of indenopyrene and benzo(ghi)perylene have not been isolated and characterized previously.

This report concludes our identification studies on the high MW PAH of cigarette smoke and complements the results on the middle region (9). Current work on the low MW, highly-alkylated PAH will be described in this journal in the near future.

\section{SUMMARY}

A gel filtration chromatography method was developed for the isolation and concentration of the high molecular weight polynuclear aromatic hydrocarbons (PAH) contained in the most biologically active fraction of cigarette smoke condensate (CSC). The unusually complex 
mixture of large PAH found in CSC necessitated the use of preparative gas chromatography followed by high-pressure liquid chromatography to achieve separation and identification. Mass spectral, ultraviolet absorption, and chromatographic retention data were needed for the comprehensive identification of the large molecular weight PAH components of CSC. The majority of the more than 200 isolated compounds were identified. Compounds newly identified in CSC included 3,4-dimethylenepyrene, 3,4-trimethylenepyrene, cyclopenta(c,d)pyrene, 4,5-methylenetriphenylene, benzo(b)perylene, and several dibenzofluoranthenes.

\section{ZUSAMMENFASSUNG}

Es wurde ein gel-diromatographisches Verfahren entwidkelt zur Isolierung und Konzentrierung der polycyclischen aromatischen Kohlenwasserstoffe (PAH) hohen Molekulargewichts, die in der biologisch am stärksten aktiven Fraktion des Cigarettenraudhondensates (CSC) enthalten sind. Die ungewöhnlich komplexe Mischung dieser im Raudkondensat gefundenen Kohlenwasserstoffe exforderte zur Erzielung von Trennung und Identifizierung präparative Gaschromatographie mit nachfolgender Hochdrudk-Flüssig-Chromatographie (HPLC). Zur umfassenden Identifizierung der im Kondensat befindlichen polycycliscten aromatischen Kohlenwasserstoffe mit hohem Molekulargewidht waren massenspektrometrische Daten, UV-Absorptionswerte und chromatographische Retentionszeiten notwendig. Von den mehr als 200 isolierten Verbindungen wurde der größte Teil identifiziert. Im Cigarettenrauchkondensat erstmalig identifizierte Verbindungen waren neben anderen 3,4Dimethylenpyren, 3,4-Trimethylenpyren, Cyclopenta(c,d)pyren, 4,5-Methylentriphenylen, Benz(b)perylen und mehrere Dibenzfluoranthene.

\section{RESUME}

On a développé une méthode de dhromatographie à perméation de gel pour l'isolation et la concentration des hydrocarbures polynucleaires aromatiques (PAH) de haut poids moléculaire, présents dans la fraction biologiquement la plus active du condensat de fumée de cigarette (CSC). Le mélange particulièrement complexe de ces PAH dans le CSC a exigé l'utilisation des techniques de dromatographie préparative en phase gazeuse suivie de chromatographie liquide à haute pression, afin de pouvoir isoler et identifier les composés. Les données de spectrographie de masse, d'absorption U.V. et de rétention chromatographique ont été requises pour l'identification complète des PAH de haut poids moléculaire du CSC. On a identifié la majorité des 200 composés isolés. Parmi les nouveaux composés identifiés dans le CSC, l'on peut citer le 3,4-diméthylène-pyrène, le 3,4triméthylène-pyrène, le cyclopenta(c,d)pyrène, le 4,5méthylènetriphénylène, le benzo(b)pérylène et plusieurs dibenzofluoranthènes.

\section{REFERENCES}

1. Stedman, R. L., R. L. Miller, L. Lakritz, and W. J. Chamberlain: Chem. and Ind. 1968, 394.

2. Swain, A. P., J. E. Cooper, R. L. Stedman, and F. G. Bodk: Beitr. Tabakforsch. 5 (1969) 109.

3. Bodk, F. G., A. P. Swain, and R. L. Stedman: J. Natl. Cancer Inst. 49 (1972) 477.

4. Swain, A. P., F. G. Bock, J. E. Cooper, W. J. Chamberlain, E. D. Strange, L. Lakritz, and R. L. Stedman: Beitr. Tabakforsch. 7 (1973) 1.

5. Chamberlain, W. J., D. B. Walters, M. E. Snook, O. T. Chortyk, and F. J. Akin: Beitr. Tabakforsch. 8 (1975) 133.

6. Akin, F. J., W. J. Chamberlain, and O. T. Chortyk: J. Natl. Cancer Inst. 54 (1975) 907.

7. Bock, F. G., A. P. Swain, and R. L. Stedman: J. Natl. Cancer Inst. 44 (1970) 1305.

8. Akin, F. J., M. E. Snook, R. F. Severson, W. J. Chamberlain, and D. B. Walters: J. Natl. Cancer Inst. 57 (1976) 191.

9. Snook, M. E., R. F. Severson, H. C. Higman, R. F. Arrendale, and $O$. 'T. Chortyk: Beitr. Tabakforsd. 8 (1976) 250.

10. Swain, A. P., J. E. Cooper, and R. L. Stedman: Cancer Res. 29 (1969) 579.

11. Severson, R. F, M. E. Snook, O. T. Chortyk, and R. F. Arrendale: Beitr. Tabakforsch. 8 (1976) 273.

12. Severson, R. F., M. E. Snook, R. F. Arrendale, and O. T. Chortyk: Anal. Chem. 48 (1976) 1866.

13. Snook, M. E.: Anal. Chim. Acta 81 (1976) 423.

14. Oro, J., and J. Han: Science 158 (1966) 1393.

15. Wynder, E. L., and D. Hoffmann: Tobacco and tobacco smoke: Studies in experimental carcinogenesis; Academic Press, New York and London, 1967.

16. Stedman, R. L.: Chem. Rev. 68 (1968) 153, and references therein.

17. Stubbs, H. W. D., and S. H. Tudker: J. Chem. Soc. 1974, 277.

18. Gold, A.: Anal. Chem. 47 (1975) 1469.

19. Clar, E. J.: Aromatische Kohlenwasserstoffe/Polycyclische Systeme; Springer-Verlag, Berlin - Göttingen - Heidelberg, 1952.

20. Lavit-Lamy, D., and N. P. Buii-Hoi: Bull. Soc. Chim. France 1966, 2613.

21. Frandk, H. G., and H. Buffleb: Justus Liebigs Ann. Chem. 701 (1967) 53.

The authors' address:

Tobacco Laboratory, Agricultural Research Service, U.S. Department of Agriculture,

P.O. Box 5677 ,

Athens, Georgia, 30604, U.S.A. 